Article

\title{
Trapezium-Type Inequalities for an Extension of Riemann-Liouville Fractional Integrals Using Raina's Special Function and Generalized Coordinate Convex Functions
}

\author{
Miguel Vivas-Cortez ${ }^{1, *,+} \oplus$, Artion Kashuri ${ }^{2,+} \oplus$, Rozana Liko ${ }^{2,+} \oplus$ and \\ Jorge Eliecer Hernández Hernández ${ }^{3,+} \mathbb{D}$ \\ 1 Escuela de Ciencias Físicas y Matemáticas, Facultad de Ciencias Exactas y Naturales, Pontificia Universidad \\ Católica del Ecuador, Av. 12 de Octubre 1076, Apartado 17-01-2184, Quito, Ecuador \\ 2 Department of Mathematics, Faculty of Technical Science, University Ismail Qemali, 1001 Vlora, Albania; \\ artion.kashuri@univlora.edu.al (A.K.); rozanaliko86@gmail.com (R.L.) \\ 3 Departamento de Técnicas Cuantitativas, Decanato de Ciencias Económicas y Empresariales, \\ Universidad Centroccidental Lisandro Alvarado, Av. 20 esq. Av Moran, Edf. Los Militares, Piso 2, \\ Barquisimeto 3001, Venezuela; jorgehernandez@ucla.edu.ve \\ * Correspondence: mjvivas@puce.edu.ec \\ + These authors contributed equally to this work.
}

Received: 15 August 2020 ; Accepted: 10 September 2020; Published: 15 October 2020

\begin{abstract}
In this paper, the authors analyse and study some recent publications about integral inequalities related to generalized convex functions of several variables and the use of extended fractional integrals. In particular, they establish a new Hermite-Hadamard inequality for generalized coordinate $\phi$-convex functions via an extension of the Riemann-Liouville fractional integral. Furthermore, an interesting identity for functions with two variables is obtained, and with the use of it, some new extensions of trapezium-type inequalities using Raina's special function via generalized coordinate $\phi$-convex functions are developed. Various special cases have been studied. At the end, a brief conclusion is given as well.
\end{abstract}

Keywords: trapezium-type inequality; generalized convexity; special functions

MSC: 52A01; 26D15; 32A17

\section{Introduction}

The application of the concept of convexity in modern analysis is a notorious fact [1-3]. Due to its importance and applications, this concept has been generalized in different ways. It is also important to mention that convex functions are closely related to certain inequalities present in different branches of science such as economics, biology, and optimization, among other [2,4,5]. Referring to the development of the concept of convexity, many authors have introduced new definitions and properties of these and have related them to the study of inequalities [6-25].

This property is defined in the following works of Jensen J.L.W.V. (1905 and 1906) [26,27] as follows.

Definition 1. A function $f: I \subseteq \mathbb{R} \longrightarrow \mathbb{R}$ is said to be convex on $I$, if:

$$
f\left((1-\imath) \ell_{1}+\imath \ell_{2}\right) \leq(1-\imath) f\left(\ell_{1}\right)+\imath f\left(\ell_{2}\right)
$$


holds for every $\ell_{1}, \ell_{2} \in I$, and $\imath \in[0,1]$.

This property is a necessary condition for the classical Hermite-Hadamard inequality, which is established as follows.

Theorem 1. Let $f: I \subseteq \mathbb{R} \longrightarrow \mathbb{R}$ be a convex function on $I$ and $\ell_{1}, \ell_{2} \in I$ with $\ell_{1}<\ell_{2}$. Then, the following inequality holds:

$$
f\left(\frac{\ell_{1}+\ell_{2}}{2}\right) \leq \frac{1}{\ell_{2}-\ell_{1}} \int_{\ell_{1}}^{\ell_{2}} f(x) d x \leq \frac{f\left(\ell_{1}\right)+f\left(\ell_{2}\right)}{2} .
$$

This inequality (6) is also known as the trapezium inequality.

Furthermore, several papers have also been published that relate integral inequalities to fractional calculus and special functions [14,28]. In [29], Sambandham, S. wrote: "the advantage of using fractional derivative versus the integer derivative is that the integer derivative is local in nature, where as the fractional derivative is global in nature"; this notion invites us to think about the behaviour of generalized convex functions in the setting of integral inequalities of fractional order.

Given the introduction of an extension of the Riemann-Liouville fractional integral made by Awan [30] and the relevance of the Hermite-Hadamard inequality in the field of statistics and probability theory, which in turn involves all the research in applied science, the purpose of the present work is to establish some integral inequalities of the trapezium type using this type of double integral for generalized convex functions in coordinates.

\section{Preliminaries}

Following the notation used by Dragomir S.S. in [8], we recall the following definition. Let us consider the rectangle $\Delta=\left[\ell_{1}, \ell_{2}\right] \times\left[\ell_{3}, \ell_{4}\right] \subset \mathbb{R}^{2}$ with $\ell_{1}<\ell_{2}$ and $\ell_{3}<\ell_{4}$. A function $f: \Delta \longrightarrow \mathbb{R}$ is said to be convex on $\Delta$ if the following inequality holds:

$$
f(\imath x+(1-\imath) z, \imath y+(1-\imath) w) \leq \imath f(x, y)+(1-\imath) f(z, w),
$$

for all $(x, y),(z, w) \in \Delta$ and $\imath \in[0,1]$. A function $f: \Delta \longrightarrow \mathbb{R}$ is said to be convex on coordinates $\Delta$ if the partial functions $f_{y}:\left[\ell_{1}, \ell_{2}\right] \longrightarrow \mathbb{R}, f_{y}(u)=f(u, y)$, and $f_{x}:\left[\ell_{3}, \ell_{4}\right] \longrightarrow \mathbb{R}, f_{x}(v)=f(x, v)$ are convex for all $x \in\left[\ell_{1}, \ell_{2}\right]$ and $y \in\left[\ell_{3}, \ell_{4}\right]$.

In the work of Awan et al. [30], the following definition used in the study of a two-dimensional extension of the Hermite-Hadamard inequality was found.

Definition 2. Consider the rectangle $\Delta=\left[\ell_{1}, \ell_{2}\right] \times\left[\ell_{3}, \ell_{4}\right] \subset \mathbb{R}^{2}$. A function $f: \Delta \longrightarrow \mathbb{R}$ is said to be coordinated convex on $\Delta$, if:

$$
\begin{aligned}
& f\left(\imath_{1} x+\left(1-\imath_{1}\right) y, \imath_{2} u+\left(1-\imath_{2}\right) w\right) \\
& \leq \imath_{1} \iota_{2} f(x, u)+\imath_{1}\left(1-\imath_{2}\right) f(x, w) \\
& +\iota_{\mathbf{2}}\left(1-\iota_{\mathbf{1}}\right) f(y, u)+\left(1-\iota_{\mathbf{1}}\right)\left(1-\iota_{\mathbf{2}}\right) f(z, w),
\end{aligned}
$$

whenever $x, y \in\left[\ell_{1}, \ell_{2}\right], u, w \in\left[\ell_{3}, \ell_{4}\right]$ and $\imath_{1}, \iota_{2} \in[0,1]$.

Dragomir in [8] extended the concept of classical convex functions on coordinates, and the Hermite-Hadamard type inequality using convex functions on coordinates was established; furthermore, M.Z. Sarikaya in [31], using the Riemann-Liouville fractional integral, extended the Hermite-Hadamard inequality for convex functions on coordinates. For other recent results, please see [32-36]. 
Noor M.A. in [16] introduced the concept of $\phi$-convex function with the assumption that $K$ is a non-empty closed set in $\mathbb{R}^{n}$ and $\phi: K \rightarrow \mathbb{R}$ a continuous function.

Definition 3. Let $u \in K$. If there exists a function $\phi$ such that the set $K$ is said to be a $\phi$-convex set:

$$
\ell_{1}+\imath e^{i \phi}\left(\ell_{2}-\ell_{1}\right) \in K
$$

for all $\ell_{1}, \ell_{2}$ in $K$ and $t \in[0,1]$, then the set $K$ is usually called a $\phi$-convex set.

Definition 4. Given a function $f: K \rightarrow \mathbb{R}$, where $K$ is $\phi$-convex set, if:

$$
f\left(\ell_{1}+\imath e^{i \phi}\left(\ell_{2}-\ell_{1}\right)\right) \leq(1-\imath) f\left(\ell_{1}\right)+\imath f\left(\ell_{2}\right), \quad \forall \ell_{1}, \ell_{2} \in K, \imath \in[0,1] .
$$

then the function is called $\phi$-convex.

The function $f$ is said to be $\phi$-concave iff $(-f)$ is $\phi$-convex. Note that every convex function is $\phi$-convex, but the converse does not hold in general.

The following class of functions, introduced by Raina R.K. in [37], is defined by:

$$
\mathcal{F}_{\rho, \lambda}^{\sigma}(z)=\mathcal{F}_{\rho, \lambda}^{\sigma(0), \sigma(1), \ldots}(z)=\sum_{k=0}^{+\infty} \frac{\sigma(k)}{\Gamma(\rho k+\lambda)} z^{k}
$$

where $\rho, \lambda>0,|z|<R$, and:

$$
\sigma=(\sigma(0), \ldots, \sigma(k), \ldots)
$$

is a bounded sequence of positive real numbers.

If we take in (2) $\rho=1, \lambda=0$, and:

$$
\sigma(k)=\frac{\left((\alpha)_{k}(\beta)_{k}\right)}{(\gamma)_{k}} \text { for } k=0,1,2, \ldots,
$$

for any parameters $\alpha, \beta$, and $\gamma$ which may be in $\mathbb{R}$ or $\mathbb{C}$ (provided that $\gamma \neq 0,-1,-2, \ldots$ ), and the symbol $(a)_{k}$ denotes the quantity:

$$
(a)_{k}=\frac{\Gamma(a+k)}{\Gamma(a)}=a(a+1) \ldots(a+k-1), \quad k=0,1,2, \ldots,
$$

and restricts its domain to $|z| \leq 1$ (with $z \in \mathbb{C}$ ), then we have the classical hypergeometric function:

$$
\mathcal{F}_{\rho, \lambda}^{\sigma}(z)=F(\alpha, \beta ; \gamma ; z)=\sum_{k=0}^{+\infty} \frac{(\alpha)_{k}(\beta)_{k}}{k !(\gamma)_{k}} z^{k}
$$

Furthermore, The classical Mittag-Leffler function defined by:

$$
E_{\alpha}(z)=\sum_{k=0}^{+\infty} \frac{1}{\Gamma(1+\alpha k)} z^{k}
$$

is obtained by the replacement of $\sigma=(1,1, \ldots)$ with $\rho=\alpha,(\operatorname{Re}(\alpha)>0), \lambda=1$ and restricting its domain to $z \in \mathbb{C}$ in (2).

Recently, Vivas-Cortez et al. in [38] introduced a class of sets and functions using Raina's function (2). 
Definition 5. Let $\rho, \lambda>0$, and $\sigma=(\sigma(0), \ldots, \sigma(k), \ldots)$ is a bounded sequence of positive real numbers. A non-empty set $K$ is said to be a generalized $\phi$-convex set, if:

$$
\ell_{1}+\imath \mathcal{F}_{\rho, \lambda}^{\sigma}\left(\ell_{2}-\ell_{1}\right) \in K, \quad \forall \ell_{1}, \ell_{2} \in K \text { and } \imath \in[0,1]
$$

and if a function $f: K \rightarrow \mathbb{R}$ satisfies the following inequality:

$$
f\left(\ell_{1}+\imath \mathcal{F}_{\rho, \lambda}^{\sigma}\left(\ell_{2}-\ell_{1}\right)\right) \leq(1-\imath) f\left(\ell_{1}\right)+\imath f\left(\ell_{2}\right),
$$

for all $\imath \in[0,1]$ and $\ell_{1}, \ell_{2} \in K$, then $f$ is called generalized $\phi$-convex, where $\mathcal{F}_{\rho, \lambda}^{\sigma}(\cdot)$ is Raina's function.

Remark 1. Taking $\rho=1, \lambda=0$ and $\sigma=(0,1,0,0, \cdots)$ in (2), then $\mathcal{F}_{\rho, \lambda}^{\sigma}\left(\ell_{2}-\ell_{1}\right)=\ell_{2}-\ell_{1}>0$, and so, we obtain Definition 1.

Using the same idea from Vivas-Cortez et al. in [38], we are in the position to introduce the generalized coordinate $\phi$-convex set and also the generalized coordinate $\phi$-convex function as follows.

Definition 6. Let $\rho_{1}, \rho_{2}, \lambda_{1}, \lambda_{2}>0$, and $\sigma_{1}=\left(\sigma_{1}(0), \ldots, \sigma_{1}(k), \ldots\right), \sigma_{2}=\left(\sigma_{2}(0), \ldots, \sigma_{2}(k), \ldots\right)$ be bounded sequences of positive real numbers. A non-empty set $K \times K \subset \mathbb{R}^{2}$ is said to be a generalized coordinate $\phi$-convex set, if:

$$
\left(\ell_{1}+\imath \mathcal{F}_{\rho_{1}, \lambda_{1}}^{\sigma_{1}}\left(\ell_{2}-\ell_{1}\right), \ell_{3}+j \mathcal{F}_{\rho_{2}, \lambda_{2}}^{\sigma_{2}}\left(\ell_{4}-\ell_{3}\right)\right) \in K \times K,
$$

for all $\ell_{1}, \ell_{2}, \ell_{3}, \ell_{4} \in K$ and $\imath_{1}, \in[0,1]$, where $\mathcal{F}_{\rho_{1}, \lambda_{1}}^{\sigma_{1}}(\cdot)$ and $\mathcal{F}_{\rho_{2}, \lambda_{2}}^{\sigma_{2}}(\cdot)$ are Raina's functions.

Definition 7. Consider the rectangle $\Delta=\left[\ell_{1}, \ell_{1}+\mathcal{F}_{\rho_{1}, \lambda_{1}}^{\sigma_{1}}\left(\ell_{2}-\ell_{1}\right)\right] \times\left[\ell_{3}, \ell_{3}+\mathcal{F}_{\rho_{2}, \lambda_{2}}^{\sigma_{2}}\left(\ell_{4}-\ell_{3}\right)\right] \subset \mathbb{R}^{2}$. A function $f: \Delta \longrightarrow \mathbb{R}$ is said to be generalized coordinate $\phi$-convex on $\Delta$, if:

$$
\begin{aligned}
f\left(x+\iota_{1} \mathcal{F}_{\rho_{1}, \lambda_{1}}^{\sigma_{1}}(x-y), u+\iota_{2}\right. & \left.\mathcal{F}_{\rho_{2}, \lambda_{2}}^{\sigma_{2}}(u-w)\right) \\
\leq & \left(1-\iota_{\mathbf{1}}\right)\left(1-\iota_{2}\right) f(x, u)+\left(1-\iota_{\mathbf{1}}\right) \iota_{\mathbf{2}} f(x, w) \\
& +\iota_{\mathbf{1}}\left(1-\iota_{\mathbf{2}}\right) f(y, u)+\iota_{\mathbf{1}} \iota_{\mathbf{2}} f(y, w),
\end{aligned}
$$

whenever $(x, u),(x, w),(y, u),(y, w) \in \Delta$ and $\imath_{1}, \iota_{2} \in[0,1]$.

Remark 2. Taking $\rho_{1}=\rho_{2}=1, \lambda_{1}=\lambda_{2}=0$, and $\sigma_{1}=(0,1,0,0, \cdots), \sigma_{2}=(0,1,0,0, \cdots)$, then $\mathcal{F}_{\rho_{1}, \lambda_{1}}^{\sigma_{1}}(x-y)=x-y$ and $\mathcal{F}_{\rho_{2}, \lambda_{2}}^{\sigma_{2}}(u-w)=u-w$ in Definition 7 , then we obtain Definition 2.

Awan M.U. et al. in [30] defined some new extensions for fractional integrals.

Definition 8. Let $f \in L_{1}\left(\left[\ell_{1}, \ell_{2}\right] \times\left[\ell_{3}, \ell_{4}\right]\right)$. The Riemann-Liouville integrals $\mathcal{J}_{\ell_{1}^{+}, \ell_{3}^{+}}^{\alpha, \beta} \mathcal{J}_{\ell_{1}^{+}, \ell_{4}^{-}}^{\alpha, \beta}, \mathcal{J}_{\ell_{2}^{-}, \ell_{3}^{+}}^{\alpha, \beta}$, and $\mathcal{J}_{\ell_{2}^{-}, \ell_{4}^{-}}^{\alpha, \beta}$ of order $\alpha, \beta>0$, where $\ell_{1}, \ell_{3} \geq 0$ and $\ell_{1}<\ell_{2}, \ell_{3}<\ell_{4}$ are defined by:

$$
\begin{array}{r}
\mathcal{J}_{\ell_{1}^{+}, \ell_{3}^{+}}^{\alpha, \beta} f(x, y)=\frac{1}{\Gamma(\alpha) \Gamma(\beta)} \int_{\ell_{1}}^{x} \int_{\ell_{3}}^{y}(x-t)^{\alpha-1}(y-s)^{\beta-1} f(t, s) d s d t, \\
\quad \text { for } x>\ell_{1}, y>\ell_{3} \\
\mathcal{J}_{\ell_{1}^{+}, \ell_{4}^{-}}^{\alpha, \beta} f(x, y)=\frac{1}{\Gamma(\alpha) \Gamma(\beta)} \int_{\ell_{1}}^{x} \int_{y}^{\ell_{4}}(x-t)^{\alpha-1}(s-y)^{\beta-1} f(t, s) d s d t, \\
\text { for } x>\ell_{1}, y<\ell_{4}
\end{array}
$$




$$
\begin{array}{r}
\mathcal{J}_{\ell_{2}^{-}, \ell_{3}^{+}}^{\alpha, \beta} f(x, y)=\frac{1}{\Gamma(\alpha) \Gamma(\beta)} \int_{x}^{\ell_{2}} \int_{\ell_{3}}^{y}(t-x)^{\alpha-1}(y-s)^{\beta-1} f(t, s) d s d t, \\
\text { for } x<\ell_{2}, y>\ell_{3} \\
\mathcal{J}_{\ell_{2}^{-}, \ell_{4}^{-}}^{\alpha, \beta} f(x, y)=\frac{1}{\Gamma(\alpha) \Gamma(\beta)} \int_{x}^{\ell_{2}} \int_{y}^{\ell_{4}}(t-x)^{\alpha-1}(s-y)^{\beta-1} f(t, s) d s d t, \\
\text { for } x<\ell_{2}, y<\ell_{4}
\end{array}
$$

and

$$
\mathcal{J}_{\ell_{1}^{+}, \ell_{3}^{+}}^{1,1} f(x, y)=\int_{\ell_{1}}^{x} \int_{\ell_{3}}^{y} f(t, s) d s d t, \text { for } x>\ell_{1}, y>\ell_{3} .
$$

From the above definition and fixing the mean value between the extremes of the intervals, we have:

$$
\begin{aligned}
& \mathcal{J}_{\ell_{1}^{+}}^{\alpha} f\left(x, \frac{\ell_{3}+\ell_{4}}{2}\right)=\frac{1}{\Gamma(\alpha)} \int_{\ell_{1}}^{x}(x-t)^{\alpha-1} f\left(t, \frac{\ell_{3}+\ell_{4}}{2}\right) d t, \quad x>\ell_{1}, \\
& \mathcal{J}_{\ell_{2}^{-}}^{\alpha} f\left(x, \frac{\ell_{3}+\ell_{4}}{2}\right)=\frac{1}{\Gamma(\alpha)} \int_{x}^{\ell_{2}}(t-x)^{\alpha-1} f\left(t, \frac{\ell_{3}+\ell_{4}}{2}\right) d t, \quad x<\ell_{2}, \\
& \mathcal{J}_{\ell_{3}^{+}}^{\beta} f\left(\frac{\ell_{1}+\ell_{2}}{2}, y\right)=\frac{1}{\Gamma(\beta)} \int_{\ell_{3}}^{y}(y-s)^{\beta-1} f\left(\frac{\ell_{1}+\ell_{2}}{2}, s\right) d s, \quad y>\ell_{3}, \\
& \mathcal{J}_{\ell_{4}^{-}}^{\beta} f\left(\frac{\ell_{1}+\ell_{2}}{2}, y\right)=\frac{1}{\Gamma(\beta)} \int_{y}^{\ell_{4}}(s-y)^{\beta-1} f\left(\frac{\ell_{1}+\ell_{2}}{2}, s\right) d s, \quad y<\ell_{4} .
\end{aligned}
$$

Motivated by the aforementioned literature, the paper is organized as follows: In Section 3, a new Hermite-Hadamard inequality for generalized functions in Definition 7 via the Riemann-Liouville fractional integral will be established. Furthermore, an interesting identity for functions with two variables will be given. By using the established identity, some new extensions of trapezium-type inequalities for Raina's fractional integral operators via generalized coordinate $\phi$-convex functions and some special cases will be obtained. In Section 4, a brief conclusion will be provided as well.

\section{Main Results}

Our first result is the Hermite-Hadamard inequality for generalized coordinate $\phi$-convex functions via the Riemann-Liouville fractional integral.

Theorem 2. Let $f: \Delta \subset \mathbb{R}^{2} \longrightarrow \mathbb{R}$ be a generalized coordinate $\phi$-convex function on $\Delta \subset \mathbb{R}^{2}$ with $\mathcal{F}_{\rho_{1}, \lambda_{1}}^{\sigma_{1}}\left(\ell_{2}-\ell_{1}\right)>0$ and $\mathcal{F}_{\rho_{2}, \lambda_{2}}^{\sigma_{2}}\left(\ell_{4}-\ell_{3}\right)>0$ and $f \in L_{1}(\Delta)$. Then, the following inequalities holds:

$$
f\left(\frac{2 \ell_{1}+\mathcal{F}_{\rho_{1}, \lambda_{1}}^{\sigma_{1}}\left(\ell_{2}-\ell_{1}\right)}{2}, \frac{2 \ell_{3}+\mathcal{F}_{\rho_{2}, \lambda_{2}}^{\sigma_{2}}\left(\ell_{4}-\ell_{3}\right)}{2}\right)
$$

$$
\leq \frac{\Gamma(\alpha+1) \Gamma(\beta+1)}{4\left(\mathcal{F}_{\rho_{1}, \lambda_{1}}^{\sigma_{1}}\left(\ell_{2}-\ell_{1}\right)\right)^{\alpha}\left(\mathcal{F}_{\rho_{2}, \lambda_{2}}^{\sigma_{2}}\left(\ell_{4}-\ell_{3}\right)\right)^{\beta}} \times
$$




$$
\begin{gathered}
{\left[J_{\left[\ell_{1}+\mathcal{F}_{\rho_{1}, \lambda_{1}}^{\sigma_{1}}\left(\ell_{2}-\ell_{1}\right)\right]^{-},\left[\ell_{3}+\mathcal{F}_{\rho_{2}, \lambda_{2}}^{\sigma_{2}}\left(\ell_{4}-\ell_{3}\right)\right]^{-}}^{\alpha} f\left(\ell_{1}, \ell_{3}\right)\right.} \\
+J_{\left[\ell_{1}+\mathcal{F}_{\rho_{1}, \lambda_{1}}^{\sigma_{1}}\left(\ell_{2}-\ell_{1}\right)\right]^{-}, \ell_{3}^{+}}^{\alpha, \beta} f\left(\ell_{1}, \ell_{3}+\mathcal{F}_{\rho_{2}, \lambda_{2}}^{\sigma_{2}}\left(\ell_{4}-\ell_{3}\right)\right) \\
+J_{\ell_{1}^{+},\left[\ell_{3}+\mathcal{F}_{\rho_{2}, \lambda_{2}}^{\sigma_{2}}\left(\ell_{4}-\ell_{3}\right)\right]^{-}}^{\alpha,} f\left(\ell_{1}+\mathcal{F}_{\rho_{1}, \lambda_{1}}^{\sigma_{1}}\left(\ell_{2}-\ell_{1}\right), \ell_{3}\right) \\
\left.+J_{\ell_{1}^{+}, \ell_{3}^{+}}^{\alpha, \beta} f\left(\ell_{1}+\mathcal{F}_{\rho_{1}, \lambda_{1}}^{\sigma_{1}}\left(\ell_{2}-\ell_{1}\right), \ell_{3}+\mathcal{F}_{\rho_{2}, \lambda_{2}}^{\sigma_{2}}\left(\ell_{4}-\ell_{3}\right)\right)\right] \\
\leq \frac{f\left(\ell_{1}, \ell_{3}\right)+f\left(\ell_{1}, \ell_{4}\right)+f\left(\ell_{2}, \ell_{3}\right)+f\left(\ell_{2}, \ell_{4}\right)}{4} .
\end{gathered}
$$

Proof. Since $f$ is a generalized coordinate $\phi$-convex function and using the change of variables $x=\ell_{1}+t_{1} \mathcal{F}_{\rho_{1}, \lambda_{1}}^{\sigma_{1}}\left(\ell_{2}-\ell_{1}\right), y=\ell_{1}+\left(1-t_{1}\right) \mathcal{F}_{\rho_{1}, \lambda_{1}}^{\sigma_{1}}\left(\ell_{2}-\ell_{1}\right), u=\ell_{3}+r_{1} \mathcal{F}_{\rho_{2}, \lambda_{2}}^{\sigma_{2}}\left(\ell_{4}-\ell_{3}\right), w=\ell_{3}+$ $\left(1-r_{1}\right) \mathcal{F}_{\rho_{2}, \lambda_{2}}^{\sigma_{2}}\left(\ell_{4}-\ell_{3}\right)$, and $t=r=\frac{1}{2}$, we have:

$$
\begin{aligned}
& f\left(\frac{2 \ell_{1}+\mathcal{F}_{\rho_{1}, \lambda_{1}}^{\sigma_{1}}\left(\ell_{2}-\ell_{1}\right)}{2}, \frac{2 \ell_{3}+\mathcal{F}_{\rho_{2}, \lambda_{2}}^{\sigma_{2}}\left(\ell_{4}-\ell_{3}\right)}{2}\right) \\
& \leq \frac{1}{4}\left[f\left(\ell_{1}+t_{1} \mathcal{F}_{\rho_{1}, \lambda_{1}}^{\sigma_{1}}\left(\ell_{2}-\ell_{1}\right), \ell_{3}+r_{1} \mathcal{F}_{\rho_{2}, \lambda_{2}}^{\sigma_{2}}\left(\ell_{4}-\ell_{3}\right)\right)\right. \\
& +f\left(\ell_{1}+t_{1} \mathcal{F}_{\rho_{1}, \lambda_{1}}^{\sigma_{1}}\left(\ell_{2}-\ell_{1}\right), \ell_{3}+\left(1-r_{1}\right) \mathcal{F}_{\rho_{2}, \lambda_{2}}^{\sigma_{2}}\left(\ell_{4}-\ell_{3}\right)\right) \\
& \quad+f\left(\ell_{1}+\left(1-t_{1}\right) \mathcal{F}_{\rho_{1}, \lambda_{1}}^{\sigma_{1}}\left(\ell_{2}-\ell_{1}\right), \ell_{3}+r_{1} \mathcal{F}_{\rho_{2}, \lambda_{2}}^{\sigma_{2}}\left(\ell_{4}-\ell_{3}\right)\right) \\
& \left.\quad+f\left(\ell_{1}+\left(1-t_{1}\right) \mathcal{F}_{\rho_{1}, \lambda_{1}}^{\sigma_{1}}\left(\ell_{2}-\ell_{1}\right), \ell_{3}+\left(1-r_{1}\right) \mathcal{F}_{\rho_{2}, \lambda_{2}}^{\sigma_{2}}\left(\ell_{4}-\ell_{3}\right)\right)\right] .
\end{aligned}
$$

Multiplying both sides of Inequality (7) by $t_{1}^{\alpha} r_{1}^{\beta}$ and integrating with respect to $\left(t_{1}, r_{1}\right)$ on $[0,1] \times[0,1]$, we get:

$$
\begin{aligned}
& \frac{1}{\alpha \beta} f(\left.\frac{2 \ell_{1}+\mathcal{F}_{\rho_{1}, \lambda_{1}}^{\sigma_{1}}\left(\ell_{2}-\ell_{1}\right)}{2}, \frac{2 \ell_{3}+\mathcal{F}_{\rho_{2}, \lambda_{2}}^{\sigma_{2}}\left(\ell_{4}-\ell_{3}\right)}{2}\right) \\
& \leq \int_{0}^{1} \int_{0}^{1} t_{1}^{\alpha-1} r_{1}^{\beta-1}\left[f\left(\ell_{1}+t_{1} \mathcal{F}_{\rho_{1}, \lambda_{1}}^{\sigma_{1}}\left(\ell_{2}-\ell_{1}\right), \ell_{3}+r_{1} \mathcal{F}_{\rho_{2}, \lambda_{2}}^{\sigma_{2}}\left(\ell_{4}-\ell_{3}\right)\right)\right. \\
& \quad+f\left(\ell_{1}+t_{1} \mathcal{F}_{\rho_{1}, \lambda_{1}}^{\sigma_{1}}\left(\ell_{2}-\ell_{1}\right), \ell_{3}+\left(1-r_{1}\right) \mathcal{F}_{\rho_{2}, \lambda_{2}}^{\sigma_{2}}\left(\ell_{4}-\ell_{3}\right)\right) \\
& \quad+f\left(\ell_{1}+\left(1-t_{1}\right) \mathcal{F}_{\rho_{1}, \lambda_{1}}^{\sigma_{1}}\left(\ell_{2}-\ell_{1}\right), \ell_{3}+r_{1} \mathcal{F}_{\rho_{2}, \lambda_{2}}^{\sigma_{2}}\left(\ell_{4}-\ell_{3}\right)\right) \\
&\left.+f\left(\ell_{1}+\left(1-t_{1}\right) \mathcal{F}_{\rho_{1}, \lambda_{1}}^{\sigma_{1}}\left(\ell_{2}-\ell_{1}\right), \ell_{3}+\left(1-r_{1}\right) \mathcal{F}_{\rho_{2}, \lambda_{2}}^{\sigma_{2}}\left(\ell_{4}-\ell_{3}\right)\right)\right] d r_{1} d t_{1} .
\end{aligned}
$$

Therefore,

$$
\begin{aligned}
& \frac{4}{\alpha \beta} f\left(\frac{2 \ell_{1}+\mathcal{F}_{\rho_{1}, \lambda_{1}}^{\sigma_{1}}\left(\ell_{2}-\ell_{1}\right)}{2}, \frac{2 \ell_{3}+\mathcal{F}_{\rho_{2}, \lambda_{2}}^{\sigma_{2}}\left(\ell_{4}-\ell_{3}\right)}{2}\right) \\
& \leq \frac{1}{\left(\mathcal{F}_{\rho_{1}, \lambda_{1}}^{\sigma_{1}}\left(\ell_{2}-\ell_{1}\right)\right)^{\alpha}\left(\mathcal{F}_{\rho_{2}, \lambda_{2}}^{\sigma_{2}}\left(\ell_{4}-\ell_{3}\right)\right)^{\beta}} \\
& \times\left[\int_{\ell_{1}}^{\ell_{1}+\mathcal{F}_{\rho_{1}, \lambda_{1}}^{\sigma_{1}}\left(\ell_{2}-\ell_{1}\right)} \int_{\ell_{3}}^{\ell_{3}+\mathcal{F}_{\rho_{2}, \lambda_{2}}^{\sigma_{2}}\left(\ell_{4}-\ell_{3}\right)}\left(x-\ell_{1}\right)^{\alpha-1}\left(y-\ell_{3}\right)^{\beta-1} f(x, y) d y d x\right.
\end{aligned}
$$




$$
\begin{aligned}
& +\int_{\ell_{1}}^{\ell_{1}+\mathcal{F}_{\rho_{1}, \lambda_{1}}^{\sigma_{1}}\left(\ell_{2}-\ell_{1}\right)} \int_{\ell_{3}}^{\ell_{3}+\mathcal{F}_{\rho_{2}, \lambda_{2}}^{\sigma_{2}}\left(\ell_{4}-\ell_{3}\right)}\left(x-\ell_{1}\right)^{\alpha-1} \times \\
& \left(\ell_{3}+\mathcal{F}_{\rho_{2}, \lambda_{2}}^{\sigma_{2}}\left(\ell_{4}-\ell_{3}\right)-y\right)^{\beta-1} f(x, y) d y d x \\
& +\int_{\ell_{1}}^{\ell_{1}+\mathcal{F}_{\rho_{1}, \lambda_{1}}^{\sigma_{1}}\left(\ell_{2}-\ell_{1}\right)} \int_{\ell_{3}}^{\ell_{3}+\mathcal{F}_{\rho_{2}, \lambda_{2}}^{\sigma_{2}}\left(\ell_{4}-\ell_{3}\right)}\left(\ell_{1}+\mathcal{F}_{\rho_{1}, \lambda_{1}}^{\sigma_{1}}\left(\ell_{2}-\ell_{1}\right)-x\right)^{\alpha-1} \times \\
& \left(y-\ell_{3}\right)^{\beta-1} f(x, y) d y d x \\
& +\int_{\ell_{1}}^{\ell_{1}+\mathcal{F}_{\rho_{1}, \lambda_{1}}^{\sigma_{1}}\left(\ell_{2}-\ell_{1}\right)} \int_{\ell_{3}}^{\ell_{3}+\mathcal{F}_{\rho_{2}, \lambda_{2}}^{\sigma_{2}}\left(\ell_{4}-\ell_{3}\right)}\left(\ell_{1}+\mathcal{F}_{\rho_{1}, \lambda_{1}}^{\sigma_{1}}\left(\ell_{2}-\ell_{1}\right)-x\right)^{\alpha-1} \\
& \left.\times\left(\ell_{3}+\mathcal{F}_{\rho_{2}, \lambda_{2}}^{\sigma_{2}}\left(\ell_{4}-\ell_{3}\right)-y\right)^{\beta-1} f(x, y) d y d x\right] .
\end{aligned}
$$

Multiplying and dividing by $\Gamma(\alpha) \Gamma(\beta)$ on the right side of Inequality (8), we get the required left-hand side of the Inequality (6). Furthermore, for $x=\ell_{1}, y=\ell_{2}, u=\ell_{3}$ and $w=\ell_{4}$ and using the definition of generalized coordinate $\phi$-convex function, we have:

$$
\begin{aligned}
& f\left(\ell_{1}+t \mathcal{F}_{\rho_{1}, \lambda_{1}}^{\sigma_{1}}\left(\ell_{2}-\ell_{1}\right), \ell_{3}+r \mathcal{F}_{\rho_{2}, \lambda_{2}}^{\sigma_{2}}\left(\ell_{4}-\ell_{3}\right)\right) \\
& \leq(1-t)(1-r) f\left(\ell_{1}, \ell_{3}\right)+(1-t) r f\left(\ell_{1}, \ell_{4}\right)+ \\
& t(1-r) f\left(\ell_{2}, \ell_{3}\right)+\operatorname{tr} f\left(\ell_{2}, \ell_{4}\right), \\
& f\left(\ell_{1}+t \mathcal{F}_{\rho_{1}, \lambda_{1}}^{\sigma_{1}}\left(\ell_{2}-\ell_{1}\right),(1-r) \ell_{3}+r \ell_{4}\right) \\
& \leq(1-t) r f\left(\ell_{1}, \ell_{3}\right)+(1-t)(1-r) f\left(\ell_{1}, \ell_{4}\right)+ \\
& \operatorname{tr} f\left(\ell_{2}, \ell_{3}\right)+t(1-r) f\left(\ell_{2}, \ell_{4}\right), \\
& f\left((1-t) \ell_{1}+t \ell_{2}, \ell_{3}+r \mathcal{F}_{\rho_{2}, \lambda_{2}}^{\sigma_{2}}\left(\ell_{4}-\ell_{3}\right)\right) \\
& \leq t(1-r) f\left(\ell_{1}, \ell_{3}\right)+\operatorname{tr} f\left(\ell_{1}, \ell_{4}\right)+ \\
& (1-t)(1-r) f\left(\ell_{2}, \ell_{3}\right)+(1-t) r f\left(\ell_{2}, \ell_{4}\right), \\
& f\left((1-t) \ell_{1}+t \ell_{2},(1-r) \ell_{3}+r \ell_{4}\right) \\
& \leq \operatorname{trf}\left(\ell_{1}, \ell_{3}\right)+t(1-r) f\left(\ell_{1}, \ell_{4}\right)+ \\
& (1-t) r f\left(\ell_{2}, \ell_{3}\right)+(1-t)(1-r) f\left(\ell_{2}, \ell_{4}\right) .
\end{aligned}
$$

Adding Inequalities (9)-(12), we obtain:

$$
\begin{gathered}
f\left(\ell_{1}+t \mathcal{F}_{\rho_{1}, \lambda_{1}}^{\sigma_{1}}\left(\ell_{2}-\ell_{1}\right), \ell_{3}+r \mathcal{F}_{\rho_{2}, \lambda_{2}}^{\sigma_{2}}\left(\ell_{4}-\ell_{3}\right)\right)+f\left(\ell_{1}+t \mathcal{F}_{\rho_{1}, \lambda_{1}}^{\sigma_{1}}\left(\ell_{2}-\ell_{1}\right),(1-r) \ell_{3}+r \ell_{4}\right) \\
+f\left((1-t) \ell_{1}+t \ell_{2}, \ell_{3}+r \mathcal{F}_{\rho_{2}, \lambda_{2}}^{\sigma_{2}}\left(\ell_{4}-\ell_{3}\right)\right)+f\left((1-t) \ell_{1}+t \ell_{2},(1-r) \ell_{3}+r \ell_{4}\right) \\
\leq f\left(\ell_{1}, \ell_{3}\right)+f\left(\ell_{1}, \ell_{4}\right)+f\left(\ell_{2}, \ell_{3}\right)+f\left(\ell_{2}, \ell_{4}\right) .
\end{gathered}
$$


Multiplying both sides of Inequality (13) by $t^{\alpha-1} r^{\beta-1}$ and integrating on $[0,1] \times[0,1]$ with respect to $(t, r)$, we get:

$$
\begin{gathered}
\int_{0}^{1} \int_{0}^{1} t^{\alpha-1} r^{\beta-1}\left[f\left(\ell_{1}+t \mathcal{F}_{\rho_{1}, \lambda_{1}}^{\sigma_{1}}\left(\ell_{2}-\ell_{1}\right), \ell_{3}+r \mathcal{F}_{\rho_{2}, \lambda_{2}}^{\sigma_{2}}\left(\ell_{4}-\ell_{3}\right)\right)\right. \\
+f\left(\ell_{1}+t \mathcal{F}_{\rho_{1}, \lambda_{1}}^{\sigma_{1}}\left(\ell_{2}-\ell_{1}\right),(1-r) \ell_{3}+r \ell_{4}\right) \\
+f\left((1-t) \ell_{1}+t \ell_{2}, \ell_{3}+r \mathcal{F}_{\rho_{2}, \lambda_{2}}^{\sigma_{2}}\left(\ell_{4}-\ell_{3}\right)\right) \\
\left.+f\left((1-t) \ell_{1}+t \ell_{2},(1-r) \ell_{3}+r \ell_{4}\right)\right] d r d t \\
\leq \int_{0}^{1} \int_{0}^{1} t^{\alpha-1} r^{\beta-1}\left(f\left(\ell_{1}, \ell_{3}\right)+f\left(\ell_{1}, \ell_{4}\right)+f\left(\ell_{2}, \ell_{3}\right)+f\left(\ell_{2}, \ell_{4}\right)\right) d r d t .
\end{gathered}
$$

This implies that:

$$
\begin{aligned}
& \frac{\Gamma(\alpha+1) \Gamma(\beta+1)}{4\left(\mathcal{F}_{\rho_{1}, \lambda_{1}}^{\sigma_{1}}\left(\ell_{2}-\ell_{1}\right)\right)^{\alpha}\left(\mathcal{F}_{\rho_{2}, \lambda_{2}}^{\sigma_{2}}\left(\ell_{4}-\ell_{3}\right)\right)^{\beta}} \times \\
& {\left[J_{\left[\ell_{1}+\mathcal{F}_{\rho_{1}, \lambda_{1}}^{\sigma_{1}}\left(\ell_{2}-\ell_{1}\right)\right]^{-},\left[\ell_{3}+\mathcal{F}_{\rho_{2}, \lambda_{2}}^{\sigma_{2}}\left(\ell_{4}-\ell_{3}\right)\right]^{-}}^{-f\left(\ell_{1}, \ell_{3}\right)}\right.} \\
& +J_{\left[\ell_{1}+\mathcal{F}_{\rho_{1}, \lambda_{1}}^{\sigma_{1}}\left(\ell_{2}-\ell_{1}\right)\right]^{-}{ }_{, \ell_{3}^{+}}}^{\alpha, \ell_{1}, \ell_{3}} f\left(\ell_{1}, \mathcal{F}_{\rho_{2}, \lambda_{2}}^{\sigma_{2}}\left(\ell_{4}-\ell_{3}\right)\right) \\
& +J_{\ell_{1}^{+},\left[\ell_{3}+\mathcal{F}_{\rho_{2}, \lambda_{2}}^{\sigma_{2}}\left(\ell_{4}-\ell_{3}\right)\right]^{-}}^{\alpha, \beta} f\left(\ell_{1}+\mathcal{F}_{\rho_{1}, \lambda_{1}}^{\sigma_{1}}\left(\ell_{2}-\ell_{1}\right), \ell_{3}\right) \\
& \left.+J_{\ell_{1}^{+}, \ell_{3}^{+}}^{\alpha, \beta} f\left(\ell_{1}+\mathcal{F}_{\rho_{1}, \lambda_{1}}^{\sigma_{1}}\left(\ell_{2}-\ell_{1}\right), \ell_{3}+\mathcal{F}_{\rho_{2}, \lambda_{2}}^{\sigma_{2}}\left(\ell_{4}-\ell_{3}\right)\right)\right] \\
& \leq \frac{f\left(\ell_{1}, \ell_{3}\right)+f\left(\ell_{1}, \ell_{4}\right)+f\left(\ell_{2}, \ell_{3}\right)+f\left(\ell_{2}, \ell_{4}\right)}{4} .
\end{aligned}
$$

Combining (8) and (14), we obtain the required right-hand side of the Inequality (6).

The proof is complete.

To derive our second results, we establish a new integral identity for the partial differentiable function involving Raina's functions.

Lemma 1. Let $f: \Delta \subset \mathbb{R}^{2} \rightarrow \mathbb{R}$ be a partial differentiable function on $\Delta$ with $\mathcal{F}_{\rho_{1}, \lambda_{1}}^{\sigma_{1}}\left(\ell_{2}-\ell_{1}\right)>0$ and $\mathcal{F}_{\rho_{2}, \lambda_{2}}^{\sigma_{2}}\left(\ell_{4}-\ell_{3}\right)>0$. If $\frac{\partial^{2} f}{\partial t \partial r} \in L_{1}(\triangle)$, then the following identity holds:

$$
\begin{gathered}
E_{f}\left(\alpha, \beta, \ell_{1}, \ell_{2}, \ell_{3}, \ell_{4} ; \mathcal{F}_{\rho_{1}, \lambda_{1}}^{\sigma_{1}}, \mathcal{F}_{\rho_{2}, \lambda_{2}}^{\sigma_{2}}\right)=\frac{\mathcal{F}_{\rho_{1}, \lambda_{1}}^{\sigma_{1}}\left(\ell_{2}-\ell_{1}\right) \mathcal{F}_{\rho_{2}, \lambda_{2}}^{\sigma_{2}}\left(\ell_{4}-\ell_{3}\right)}{4} \\
\times\left\{\int_{0}^{1} \int_{0}^{1} t^{\alpha} r^{\beta} \frac{\partial^{2} f}{\partial t \partial r}\left(\ell_{1}+t \mathcal{F}_{\rho_{1}, \lambda_{1}}^{\sigma_{1}}\left(\ell_{2}-\ell_{1}\right), \ell_{3}+r \mathcal{F}_{\rho_{2}, \lambda_{2}}^{\sigma_{2}}\left(\ell_{4}-\ell_{3}\right)\right) d r d t\right. \\
-\int_{0}^{1} \int_{0}^{1}(1-t)^{\alpha} r^{\beta} \frac{\partial^{2} f}{\partial t \partial r}\left(\ell_{1}+t \mathcal{F}_{\rho_{1}, \lambda_{1}}^{\sigma_{1}}\left(\ell_{2}-\ell_{1}\right), \ell_{3}+r \mathcal{F}_{\rho_{2}, \lambda_{2}}^{\sigma_{2}}\left(\ell_{4}-\ell_{3}\right)\right) d r d t \\
-\int_{0}^{1} \int_{0}^{1} t^{\alpha}(1-r)^{\beta} \frac{\partial^{2} f}{\partial t \partial r}\left(\ell_{1}+t \mathcal{F}_{\rho_{1}, \lambda_{1}}^{\sigma_{1}}\left(\ell_{2}-\ell_{1}\right), \ell_{3}+r \mathcal{F}_{\rho_{2}, \lambda_{2}}^{\sigma_{2}}\left(\ell_{4}-\ell_{3}\right)\right) d r d t \\
\left.+\int_{0}^{1} \int_{0}^{1}(1-t)^{\alpha}(1-r)^{\beta} \frac{\partial^{2} f}{\partial t \partial r}\left(\ell_{1}+t \mathcal{F}_{\rho_{1}, \lambda_{1}}^{\sigma_{1}}\left(\ell_{2}-\ell_{1}\right), \ell_{3}+r \mathcal{F}_{\rho_{2}, \lambda_{2}}^{\sigma_{2}}\left(\ell_{4}-\ell_{3}\right)\right) d r d t\right\},
\end{gathered}
$$


where:

$$
\begin{aligned}
& E_{f}\left(\alpha, \beta, \ell_{1}, \ell_{2}, \ell_{3}, \ell_{4} ; \mathcal{F}_{\rho_{1}, \lambda_{1}}^{\sigma_{1}}, \mathcal{F}_{\rho_{2}, \lambda_{2}}^{\sigma_{2}}\right) \\
& =\frac{f\left(\ell_{1}+\mathcal{F}_{\rho_{1}, \lambda_{1}}^{\sigma_{1}}\left(\ell_{2}-\ell_{1}\right), \ell_{3}+\mathcal{F}_{\rho_{2}, \lambda_{2}}^{\sigma_{2}}\left(\ell_{4}-\ell_{3}\right)\right)}{4}+\frac{f\left(\ell_{1}, \ell_{3}+\mathcal{F}_{\rho_{2}, \lambda_{2}}^{\sigma_{2}}\left(\ell_{4}-\ell_{3}\right)\right)}{4} \\
& +\frac{f\left(\ell_{1}+\mathcal{F}_{\rho_{1}, \lambda_{1}}^{\sigma_{1}}\left(\ell_{2}-\ell_{1}\right), \ell_{3}\right)}{4}+\frac{f\left(\ell_{1}, \ell_{3}\right)}{4} \\
& -\frac{\Gamma(\beta+1)}{4\left(\mathcal{F}_{\rho_{2}, \lambda_{2}}^{\sigma_{2}}\left(\ell_{4}-\ell_{3}\right)\right)^{\beta}} \times \\
& \times\left[J_{\left[\ell_{3}+\mathcal{F}_{\rho_{2}, \lambda_{2}}^{\sigma_{2}}\left(\ell_{4}-\ell_{3}\right)\right.}^{\beta}\right]^{-} f\left(\ell_{1}+\mathcal{F}_{\rho_{1}, \lambda_{1}}^{\sigma_{1}}\left(\ell_{2}-\ell_{1}\right), \ell_{3}\right)+J_{\left[\ell_{3}+\mathcal{F}_{\rho_{2}, \lambda_{2}}^{\sigma_{2}}\left(\ell_{4}-\ell_{3}\right)\right]^{-}}^{\beta} f\left(\ell_{1}, \ell_{3}\right) \\
& \left.+J_{\ell_{3}^{+}}^{\beta} f\left(\ell_{1}+\mathcal{F}_{\rho_{1}, \lambda_{1}}^{\sigma_{1}}\left(\ell_{2}-\ell_{1}\right), \ell_{3}+\mathcal{F}_{\rho_{2}, \lambda_{2}}^{\sigma_{2}}\left(\ell_{4}-\ell_{3}\right)\right)+J_{\ell_{3}^{+}}^{\beta} f\left(\ell_{1}, \ell_{3}+\mathcal{F}_{\rho_{2}, \lambda_{2}}^{\sigma_{2}}\left(\ell_{4}-\ell_{3}\right)\right)\right] \\
& -\frac{\Gamma(\alpha+1)}{4\left(\mathcal{F}_{\rho_{1}, \lambda_{1}}^{\sigma_{1}}\left(\ell_{2}-\ell_{1}\right)\right)^{\alpha}} \times
\end{aligned}
$$

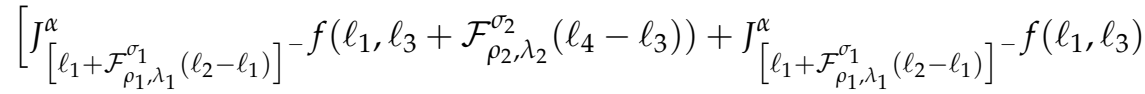

$$
\begin{aligned}
& \left.+J_{\ell_{1}^{+}}^{\alpha} f\left(\ell_{1}+\mathcal{F}_{\rho_{1}, \lambda_{1}}^{\sigma_{1}}\left(\ell_{2}-\ell_{1}\right), \ell_{3}+\mathcal{F}_{\rho_{2}, \lambda_{2}}^{\sigma_{2}}\left(\ell_{4}-\ell_{3}\right)\right)+J_{\ell_{1}^{+}}^{\alpha} f\left(\ell_{1}+\mathcal{F}_{\rho_{1}, \lambda_{1}}^{\sigma_{1}}\left(\ell_{2}-\ell_{1}\right), \ell_{3}\right)\right] \\
& +\frac{\Gamma(\alpha+1) \Gamma(\beta+1)}{4\left(\mathcal{F}_{\rho_{1}, \lambda_{1}}^{\sigma_{1}}\left(\ell_{2}-\ell_{1}\right)\right)^{\alpha-1}\left(\mathcal{F}_{\rho_{2}, \lambda_{2}}^{\sigma_{2}}\left(\ell_{4}-\ell_{3}\right)\right)^{\beta-1}} \times \\
& {\left[J_{\left[\ell_{1}+\mathcal{F}_{\rho_{1}, \lambda_{1}}^{\sigma_{1}}\left(\ell_{2}-\ell_{1}\right)\right]^{-},\left[\ell_{3}+\mathcal{F}_{\rho_{2}, \lambda_{2}}^{\sigma_{2}}\left(\ell_{4}-\ell_{3}\right)\right]^{-}} f\left(\ell_{1}, \ell_{3}\right)\right.} \\
& +J_{\ell_{1}^{+},\left[\ell_{3}+\mathcal{F}_{\rho_{2}, \lambda_{2}}^{\sigma_{2}}\left(\ell_{4}-\ell_{3}\right)\right]^{-}}^{\alpha,\left(\ell_{1}+\mathcal{F}_{\rho_{1}, \lambda_{1}}^{\sigma_{1}}\left(\ell_{2}-\ell_{1}\right), \ell_{3}\right)} \\
& +J_{\left[\ell_{1}+\mathcal{F}_{\rho_{1}, \lambda_{1}}^{\sigma_{1}}\left(\ell_{2}-\ell_{1}\right)\right]^{-}, \ell_{3}^{+}}^{\alpha, \beta} f\left(\ell_{1}, \ell_{3}+\mathcal{F}_{\rho_{2}, \lambda_{2}}^{\sigma_{2}}\left(\ell_{4}-\ell_{3}\right)\right)+ \\
& \left.J_{\ell_{1}^{+}, \ell_{3}^{+}}^{\alpha, \beta} f\left(\ell_{1}+\mathcal{F}_{\rho_{1}, \lambda_{1}}^{\sigma_{1}}\left(\ell_{2}-\ell_{1}\right), \ell_{3}+\mathcal{F}_{\rho_{2}, \lambda_{2}}^{\sigma_{2}}\left(\ell_{4}-\ell_{3}\right)\right)\right] .
\end{aligned}
$$

Proof. Let:

$$
\begin{gathered}
I_{1}=\int_{0}^{1} \int_{0}^{1} t^{\alpha} r^{\beta} \frac{\partial^{2} f}{\partial t \partial r}\left(\ell_{1}+t \mathcal{F}_{\rho_{1}, \lambda_{1}}^{\sigma_{1}}\left(\ell_{2}-\ell_{1}\right), \ell_{3}+r \mathcal{F}_{\rho_{2}, \lambda_{2}}^{\sigma_{2}}\left(\ell_{4}-\ell_{3}\right)\right) d r d t \\
=\int_{0}^{1} r^{\beta}\left[\left.\frac{t^{\alpha}}{\mathcal{F}_{\rho_{1}, \lambda_{1}}^{\sigma_{1}}\left(\ell_{2}-\ell_{1}\right)} \frac{\partial f}{\partial r}\left(\ell_{1}+t \mathcal{F}_{\rho_{1}, \lambda_{1}}^{\sigma_{1}}\left(\ell_{2}-\ell_{1}\right), \ell_{3}+r \mathcal{F}_{\rho_{2}, \lambda_{2}}^{\sigma_{2}}\left(\ell_{4}-\ell_{3}\right)\right)\right|_{0} ^{1}\right. \\
\left.-\frac{\alpha}{\mathcal{F}_{\rho_{1}, \lambda_{1}}^{\sigma_{1}}\left(\ell_{2}-\ell_{1}\right)} \int_{0}^{1} \frac{\partial f}{\partial r}\left(\ell_{1}+t \mathcal{F}_{\rho_{1}, \lambda_{1}}^{\sigma_{1}}\left(\ell_{2}-\ell_{1}\right), \ell_{3}+r \mathcal{F}_{\rho_{2}, \lambda_{2}}^{\sigma_{2}}\left(\ell_{4}-\ell_{3}\right)\right) t^{\alpha-1}\right] d r \\
=\frac{1}{\mathcal{F}_{\rho_{1}, \lambda_{1}}^{\sigma_{1}}\left(\ell_{2}-\ell_{1}\right) \mathcal{F}_{\rho_{2}, \lambda_{2}}^{\sigma_{2}}\left(\ell_{4}-\ell_{3}\right)} f\left(\ell_{1}+\mathcal{F}_{\rho_{1}, \lambda_{1}}^{\sigma_{1}}\left(\ell_{2}-\ell_{1}\right), \ell_{3}+\mathcal{F}_{\rho_{2}, \lambda_{2}}^{\sigma_{2}}\left(\ell_{4}-\ell_{3}\right)\right)
\end{gathered}
$$




$$
\begin{aligned}
& -\frac{\beta}{\mathcal{F}_{\rho_{1}, \lambda_{1}}^{\sigma_{1}}\left(\ell_{2}-\ell_{1}\right) \mathcal{F}_{\rho_{2}, \lambda_{2}}^{\sigma_{2}}\left(\ell_{4}-\ell_{3}\right)} \times \\
& \int_{0}^{1} r^{\beta-1} f\left(\ell_{1}+\mathcal{F}_{\rho_{1}, \lambda_{1}}^{\sigma_{1}}\left(\ell_{2}-\ell_{1}\right), \ell_{3}+r \mathcal{F}_{\rho_{2}, \lambda_{2}}^{\sigma_{2}}\left(\ell_{4}-\ell_{3}\right)\right) d r \\
& -\frac{\alpha}{\mathcal{F}_{\rho_{1}, \lambda_{1}}^{\sigma_{1}}\left(\ell_{2}-\ell_{1}\right) \mathcal{F}_{\rho_{2}, \lambda_{2}}^{\sigma_{2}}\left(\ell_{4}-\ell_{3}\right)} \times \\
& +\frac{\alpha \beta}{\mathcal{F}_{\rho_{1}, \lambda_{1}}^{\sigma_{1}}\left(\ell_{2}-\ell_{1}\right) \mathcal{F}_{\rho_{2}, \lambda_{2}}^{\sigma_{2}}\left(\ell_{4}-\ell_{3}\right)} f\left(\ell_{1}+t \mathcal{F}_{\rho_{1}, \lambda_{1}}^{\sigma_{1}}\left(\ell_{2}-\ell_{1}\right), \ell_{3}+\mathcal{F}_{\rho_{2}, \lambda_{2}}^{\sigma_{2}}\left(\ell_{4}-\ell_{3}\right)\right) d t \\
& \int_{0}^{1} \int_{0}^{1} t^{\alpha-1} r^{\beta-1} f\left(\ell_{1}+t \mathcal{F}_{\rho_{1}, \lambda_{1}}^{\sigma_{1}}\left(\ell_{2}-\ell_{1}\right), \ell_{3}+r \mathcal{F}_{\rho_{2}, \lambda_{2}}^{\sigma_{2}}\left(\ell_{4}-\ell_{3}\right)\right) d r d t .
\end{aligned}
$$

Similarly, we have:

$$
\begin{aligned}
& I_{2}=\int_{0}^{1} \int_{0}^{1}(1-t)^{\alpha} r^{\beta} \frac{\partial^{2} f}{\partial t \partial r}\left(\ell_{1}+t \mathcal{F}_{\rho_{1}, \lambda_{1}}^{\sigma_{1}}\left(\ell_{2}-\ell_{1}\right), \ell_{3}+r \mathcal{F}_{\rho_{2}, \lambda_{2}}^{\sigma_{2}}\left(\ell_{4}-\ell_{3}\right)\right) d r d t \\
& =-\frac{1}{\mathcal{F}_{\rho_{1}, \lambda_{1}}^{\sigma_{1}}\left(\ell_{2}-\ell_{1}\right) \mathcal{F}_{\rho_{2}, \lambda_{2}}^{\sigma_{2}}\left(\ell_{4}-\ell_{3}\right)} f\left(\ell_{1}, \ell_{3}+\mathcal{F}_{\rho_{2}, \lambda_{2}}^{\sigma_{2}}\left(\ell_{4}-\ell_{3}\right)\right) \\
& +\frac{\beta}{\mathcal{F}_{\rho_{1}, \lambda_{1}}^{\sigma_{1}}\left(\ell_{2}-\ell_{1}\right) \mathcal{F}_{\rho_{2}, \lambda_{2}}^{\sigma_{2}}\left(\ell_{4}-\ell_{3}\right)} \int_{0}^{1} r^{\beta-1} f\left(\ell_{1}, \ell_{3}+r \mathcal{F}_{\rho_{2}, \lambda_{2}}^{\sigma_{2}}\left(\ell_{4}-\ell_{3}\right)\right) d r \\
& +\frac{\alpha}{\mathcal{F}_{\rho_{1}, \lambda_{1}}^{\sigma_{1}}\left(\ell_{2}-\ell_{1}\right) \mathcal{F}_{\rho_{2}, \lambda_{2}}^{\sigma_{2}}\left(\ell_{4}-\ell_{3}\right)} \times \\
& \int_{0}^{1}(1-t)^{\alpha-1} f\left(\ell_{1}+t \mathcal{F}_{\rho_{1}, \lambda_{1}}^{\sigma_{1}}\left(\ell_{2}-\ell_{1}\right), \ell_{3}+\mathcal{F}_{\rho_{2}, \lambda_{2}}^{\sigma_{2}}\left(\ell_{4}-\ell_{3}\right)\right) d t \\
& -\frac{\alpha \beta}{\mathcal{F}_{\rho_{1}, \lambda_{1}}^{\sigma_{1}}\left(\ell_{2}-\ell_{1}\right) \mathcal{F}_{\rho_{2}, \lambda_{2}}^{\sigma_{2}}\left(\ell_{4}-\ell_{3}\right)} \times \\
& \int_{0}^{1} \int_{0}^{1}(1-t)^{\alpha-1} r^{\beta-1} f\left(\ell_{1}+t \mathcal{F}_{\rho_{1}, \lambda_{1}}^{\sigma_{1}}\left(\ell_{2}-\ell_{1}\right), \ell_{3}+r \mathcal{F}_{\rho_{2}, \lambda_{2}}^{\sigma_{2}}\left(\ell_{4}-\ell_{3}\right)\right) d r d t, \\
& I_{3}=\int_{0}^{1} \int_{0}^{1} t^{\alpha}(1-r)^{\beta} \frac{\partial^{2} f}{\partial t \partial r}\left(\ell_{1}+t \mathcal{F}_{\rho_{1}, \lambda_{1}}^{\sigma_{1}}\left(\ell_{2}-\ell_{1}\right), \ell_{3}+r \mathcal{F}_{\rho_{2}, \lambda_{2}}^{\sigma_{2}}\left(\ell_{4}-\ell_{3}\right)\right) d r d t \\
& =-\frac{1}{\mathcal{F}_{\rho_{1}, \lambda_{1}}^{\sigma_{1}}\left(\ell_{2}-\ell_{1}\right) \mathcal{F}_{\rho_{2}, \lambda_{2}}^{\sigma_{2}}\left(\ell_{4}-\ell_{3}\right)} f\left(\ell_{1}+\mathcal{F}_{\rho_{1}, \lambda_{1}}^{\sigma_{1}}\left(\ell_{2}-\ell_{1}\right), \ell_{3}\right) \\
& +\frac{\beta}{\mathcal{F}_{\rho_{1}, \lambda_{1}}^{\sigma_{1}}\left(\ell_{2}-\ell_{1}\right) \mathcal{F}_{\rho_{2}, \lambda_{2}}^{\sigma_{2}}\left(\ell_{4}-\ell_{3}\right)} \times \\
& \int_{0}^{1}(1-r)^{\beta-1} f\left(\ell_{1}+\mathcal{F}_{\rho_{1}, \lambda_{1}}^{\sigma_{1}}\left(\ell_{2}-\ell_{1}\right), \ell_{3}+r \mathcal{F}_{\rho_{2}, \lambda_{2}}^{\sigma_{2}}\left(\ell_{4}-\ell_{3}\right)\right) d r \\
& +\frac{\alpha}{\mathcal{F}_{\rho_{1}, \lambda_{1}}^{\sigma_{1}}\left(\ell_{2}-\ell_{1}\right) \mathcal{F}_{\rho_{2}, \lambda_{2}}^{\sigma_{2}}\left(\ell_{4}-\ell_{3}\right)} \int_{0}^{1} t^{\alpha-1} f\left(\ell_{1}+\mathcal{F}_{\rho_{1}, \lambda_{1}}^{\sigma_{1}}\left(\ell_{2}-\ell_{1}\right), \ell_{3}\right) d t \\
& -\frac{\alpha \beta}{\mathcal{F}_{\rho_{1}, \lambda_{1}}^{\sigma_{1}}\left(\ell_{2}-\ell_{1}\right) \mathcal{F}_{\rho_{2}, \lambda_{2}}^{\sigma_{2}}\left(\ell_{4}-\ell_{3}\right)} \times
\end{aligned}
$$




$$
\int_{0}^{1} \int_{0}^{1} t^{\alpha-1}(1-r)^{\beta-1} f\left(\ell_{1}+t \mathcal{F}_{\rho_{1}, \lambda_{1}}^{\sigma_{1}}\left(\ell_{2}-\ell_{1}\right), \ell_{3}+r \mathcal{F}_{\rho_{2}, \lambda_{2}}^{\sigma_{2}}\left(\ell_{4}-\ell_{3}\right)\right) d r d t
$$

and:

$$
\begin{aligned}
I_{4}= & \int_{0}^{1} \int_{0}^{1}(1-t)^{\alpha}(1-r)^{\beta} \frac{\partial^{2} f}{\partial t \partial r}\left(\ell_{1}+t \mathcal{F}_{\rho_{1}, \lambda_{1}}^{\sigma_{1}}\left(\ell_{2}-\ell_{1}\right), \ell_{3}+r \mathcal{F}_{\rho_{2}, \lambda_{2}}^{\sigma_{2}}\left(\ell_{4}-\ell_{3}\right)\right) d r d t \\
= & \frac{\alpha}{\mathcal{F}_{\rho_{1}, \lambda_{1}}^{\sigma_{1}}\left(\ell_{2}-\ell_{1}\right) \mathcal{F}_{\rho_{2}, \lambda_{2}}^{\sigma_{2}}\left(\ell_{4}-\ell_{3}\right)} f\left(\ell_{1}, \ell_{3}\right) \\
& -\frac{\alpha}{\mathcal{F}_{\rho_{1}, \lambda_{1}}^{\sigma_{1}}\left(\ell_{2}-\ell_{1}\right) \mathcal{F}_{\rho_{2}, \lambda_{2}}^{\sigma_{2}}\left(\ell_{4}-\ell_{3}\right)} \int_{0}^{1}(1-t)^{\alpha-1} f\left(\ell_{1}+t \mathcal{F}_{\rho_{1}, \lambda_{1}}^{\sigma_{1}}\left(\ell_{2}-\ell_{1}\right), \ell_{3}\right) d t \\
& -\frac{\beta}{\mathcal{F}_{\rho_{1}, \lambda_{1}}^{\sigma_{1}}\left(\ell_{2}-\ell_{1}\right) \mathcal{F}_{\rho_{2}, \lambda_{2}}^{\sigma_{2}}\left(\ell_{4}-\ell_{3}\right)} \int_{0}^{1}(1-r)^{\beta-1} f\left(\ell_{1}, \ell_{3}+r \mathcal{F}_{\rho_{2}, \lambda_{2}}^{\sigma_{2}}\left(\ell_{4}-\ell_{3}\right)\right) d r \\
& +\frac{\alpha \beta}{\mathcal{F}_{\rho_{1}, \lambda_{1}}^{\sigma_{1}}\left(\ell_{2}-\ell_{1}\right) \mathcal{F}_{\rho_{2}, \lambda_{2}}^{\sigma_{2}}\left(\ell_{4}-\ell_{3}\right)} \times \\
& \int_{0}^{1} \int_{0}^{1}(1-t)^{\alpha-1}(1-r)^{\beta-1} f\left(\ell_{1}+t \mathcal{F}_{\rho_{1}, \lambda_{1}}^{\sigma_{1}}\left(\ell_{2}-\ell_{1}\right), \ell_{3}+r \mathcal{F}_{\rho_{2}, \lambda_{2}}^{\sigma_{2}}\left(\ell_{4}-\ell_{3}\right)\right) d r d t .
\end{aligned}
$$

By using the change of variables, we have:

$$
\begin{aligned}
& I_{1}-I_{2}-I_{3}+I_{4} \\
& =\frac{f\left(\ell_{1}+\mathcal{F}_{\rho_{1}, \lambda_{1}}^{\sigma_{1}}\left(\ell_{2}-\ell_{1}\right), \ell_{3}+\mathcal{F}_{\rho_{2}, \lambda_{2}}^{\sigma_{2}}\left(\ell_{4}-\ell_{3}\right)\right)+f\left(\ell_{1}, \ell_{3}+r \mathcal{F}_{\rho_{2}, \lambda_{2}}^{\sigma_{2}}\left(\ell_{4}-\ell_{3}\right)\right)}{\mathcal{F}_{\rho_{1}, \lambda_{1}}^{\sigma_{1}}\left(\ell_{2}-\ell_{1}\right) \mathcal{F}_{\rho_{2}, \lambda_{2}}^{\sigma_{2}}\left(\ell_{4}-\ell_{3}\right)} \\
& +\frac{f\left(\ell_{1}+\mathcal{F}_{\rho_{1}, \lambda_{1}}^{\sigma_{1}}\left(\ell_{2}-\ell_{1}\right), \ell_{3}+f\left(\ell_{1}+, \ell_{3}\right)\right)}{\mathcal{F}_{\rho_{1}, \lambda_{1}}^{\sigma_{1}}\left(\ell_{2}-\ell_{1}\right) \mathcal{F}_{\rho_{2}, \lambda_{2}}^{\sigma_{2}}\left(\ell_{4}-\ell_{3}\right)} \\
& -\frac{\Gamma(\beta+1)}{\mathcal{F}_{\rho_{1}, \lambda_{1}}^{\sigma_{1}}\left(\ell_{2}-\ell_{1}\right)\left(\mathcal{F}_{\rho_{2}, \lambda_{2}}^{\sigma_{2}}\left(\ell_{4}-\ell_{3}\right)\right)^{\beta+1}} \times \\
& {\left[J_{\left[\ell_{3}+\mathcal{F}_{\rho_{2}, \lambda_{2}}^{\sigma_{2}}\left(\ell_{4}-\ell_{3}\right)\right]^{-}}^{\beta} f\left(\ell_{1}+\mathcal{F}_{\rho_{1}, \lambda_{1}}^{\sigma_{1}}\left(\ell_{2}-\ell_{1}\right), \ell_{3}\right)+J_{\left[\ell_{3}+\mathcal{F}_{\rho_{2}, \lambda_{2}}^{\sigma_{2}}\left(\ell_{4}-\ell_{3}\right)\right]^{-}}^{\beta} f\left(\ell_{1}, \ell_{3}\right)\right.} \\
& \left.+J_{\ell_{3}^{+}}^{\beta} f\left(\ell_{1}+\mathcal{F}_{\rho_{1}, \lambda_{1}}^{\sigma_{1}}\left(\ell_{2}-\ell_{1}\right), \ell_{3}+\mathcal{F}_{\rho_{2}, \lambda_{2}}^{\sigma_{2}}\left(\ell_{4}-\ell_{3}\right)\right)+J_{\ell_{3}^{+}}^{\beta} f\left(\ell_{1}, \ell_{3}+\mathcal{F}_{\rho_{2}, \lambda_{2}}^{\sigma_{2}}\left(\ell_{4}-\ell_{3}\right)\right)\right] \\
& -\frac{\Gamma(\alpha+1)}{\left(\mathcal{F}_{\rho_{1}, \lambda_{1}}^{\sigma_{1}}\left(\ell_{2}-\ell_{1}\right)\right)^{\alpha+1} \mathcal{F}_{\rho_{2}, \lambda_{2}}^{\sigma_{2}}\left(\ell_{4}-\ell_{3}\right)} \times \\
& {\left[J_{\left[\ell_{1}+\mathcal{F}_{\rho_{1}, \lambda_{1}}^{\sigma_{1}}\left(\ell_{2}-\ell_{1}\right)\right.}^{\alpha} f\left(\ell_{1}, \ell_{3}+\mathcal{F}_{\rho_{2}, \lambda_{2}}^{\sigma_{2}}\left(\ell_{4}-\ell_{3}\right)\right)\right.} \\
& +J_{\ell_{1}^{+}}^{\alpha} f\left(\ell_{1}+\mathcal{F}_{\rho_{1}, \lambda_{1}}^{\sigma_{1}}\left(\ell_{2}-\ell_{1}\right), \ell_{3}+\mathcal{F}_{\rho_{2}, \lambda_{2}}^{\sigma_{2}}\left(\ell_{4}-\ell_{3}\right)\right) \\
& \left.\left.+J_{\left[\ell_{1}+\mathcal{F}_{\rho_{1}, \lambda_{1}}^{\sigma_{1}}\left(\ell_{2}-\ell_{1}\right)\right.}^{\alpha}\right]^{-} f\left(\ell_{1}, \ell_{3}\right)+J_{\ell_{1}^{+}}^{\alpha} f\left(\ell_{1}+\mathcal{F}_{\rho_{1}, \lambda_{1}}^{\sigma_{1}}\left(\ell_{2}-\ell_{1}\right), \ell_{3}\right)\right] \\
& +\frac{\Gamma(\alpha+1) \Gamma(\beta+1)}{\left(\mathcal{F}_{\rho_{1}, \lambda_{1}}^{\sigma_{1}}\left(\ell_{2}-\ell_{1}\right)\right)^{\alpha}\left(\mathcal{F}_{\rho_{2}, \lambda_{2}}^{\sigma_{2}}\left(\ell_{4}-\ell_{3}\right)\right)^{\beta}} \times \\
& {\left[J_{\left[\ell_{1}+\mathcal{F}_{\rho_{1}, \lambda_{1}}^{\sigma_{1}}\left(\ell_{2}-\ell_{1}\right)\right.}^{\sigma_{1}}\right]^{-},\left[\ell_{3}+\mathcal{F}_{\rho_{2}, \lambda_{2}}^{\sigma_{2}}\left(\ell_{4}-\ell_{3}\right)\right]^{-} f\left(\ell_{1}, \ell_{3}\right)} \\
& +J_{\ell_{1}^{+},\left[\ell_{3}+\mathcal{F}_{\rho_{2}, \lambda_{2}}^{\sigma_{2}}\left(\ell_{4}-\ell_{3}\right)\right]^{-}}^{\alpha, \beta}\left(\ell_{1}+\mathcal{F}_{\rho_{1}, \lambda_{1}}^{\sigma_{1}}\left(\ell_{2}-\ell_{1}, \ell_{3}\right)\right)
\end{aligned}
$$




$$
\begin{aligned}
& +J_{\left[\ell_{1}+\mathcal{F}_{\rho_{1}, \lambda_{1}}^{\sigma_{1}}\left(\ell_{2}-\ell_{1}\right)\right]^{-}, \ell_{3}^{+}}^{\alpha, \beta} f\left(\ell_{1}, \ell_{3}+\mathcal{F}_{\rho_{2}, \lambda_{2}}^{\sigma_{2}}\left(\ell_{4}-\ell_{3}\right)\right) \\
& \left.\quad+J_{\ell_{1}^{+}, \ell_{3}^{+}}^{\alpha, \beta} f\left(\ell_{1}+\mathcal{F}_{\rho_{1}, \lambda_{1}}^{\sigma_{1}}\left(\ell_{2}-\ell_{1}\right), \ell_{3}+\mathcal{F}_{\rho_{2}, \lambda_{2}}^{\sigma_{2}}\left(\ell_{4}-\ell_{3}\right)\right)\right] .
\end{aligned}
$$

Multiplying Equality (16) by $\frac{\mathcal{F}_{\rho_{1}, \lambda_{1}}^{\sigma_{1}}\left(\ell_{2}-\ell_{1}\right) \mathcal{F}_{\rho_{2}, \lambda_{2}}^{\sigma_{2}}\left(\ell_{4}-\ell_{3}\right)}{4}$, we get the desired equality (15).

The proof is complete.

Using Lemma 1, we can derive the following theorems for generalized coordinate $\phi$-convex functions.

Theorem 3. Let $f: \Delta \subset \mathbb{R}^{2} \longrightarrow \mathbb{R}$ be a partial differentiable function on $\Delta$ with $\mathcal{F}_{\rho_{1}, \lambda_{1}}^{\sigma_{1}}\left(\ell_{2}-\ell_{1}\right)>0$ and $\mathcal{F}_{\rho_{2}, \lambda_{2}}^{\sigma_{2}}\left(\ell_{4}-\ell_{3}\right)>0$ and $\frac{\partial^{2} f}{\partial t \partial r} \in L_{1}(\Delta)$. If $\left|\frac{\partial^{2} f}{\partial t \partial r}\right|^{q}$ is a generalized coordinated $\phi$-convex function where $q>1$ and $\frac{1}{p}+\frac{1}{q}=1$, then the following inequality holds:

$$
\begin{gathered}
\left|E_{f}\left(\alpha, \beta, \ell_{1}, \ell_{2}, \ell_{3}, \ell_{4} ; \mathcal{F}_{\rho_{1}, \lambda_{1}}^{\sigma_{1}}, \mathcal{F}_{\rho_{2}, \lambda_{2}}^{\sigma_{2}}\right)\right| \leq \frac{\mathcal{F}_{\rho_{1}, \lambda_{1}}^{\sigma_{1}}\left(\ell_{2}-\ell_{1}\right) \mathcal{F}_{\rho_{2}, \lambda_{2}}^{\sigma_{2}}\left(\ell_{4}-\ell_{3}\right)}{\sqrt[p]{(p \alpha+1)(p \beta+1)}} \times \\
\sqrt[q]{\frac{\left|\frac{\partial^{2} f}{\partial t \partial r}\left(\ell_{1}, \ell_{3}\right)\right|^{q}+\left|\frac{\partial^{2} f}{\partial t \partial r}\left(\ell_{2}, \ell_{3}\right)\right|^{q}+\left|\frac{\partial^{2} f}{\partial t \partial r}\left(\ell_{1}, \ell_{4}\right)\right|^{q}+\left|\frac{\partial^{2} f}{\partial t \partial r}\left(\ell_{2}, \ell_{4}\right)\right|^{q}}{4}} .
\end{gathered}
$$

Proof. Using Lemma 1, the fact that $\left|\frac{\partial^{2} f}{\partial t \partial r}\right|^{q}$ is a generalized coordinated $\phi$-convex function, and Hölder's inequality, we have:

$$
\begin{aligned}
\mid & E_{f}\left(\alpha, \beta, \ell_{1}, \ell_{2}, \ell_{3}, \ell_{4} ; \mathcal{F}_{\rho_{1}, \lambda_{1}}^{\sigma_{1}}, \mathcal{F}_{\rho_{2}, \lambda_{2}}^{\sigma_{2}}\right) \mid \leq \frac{\mathcal{F}_{\rho_{1}, \lambda_{1}}^{\sigma_{1}}\left(\ell_{2}-\ell_{1}\right) \mathcal{F}_{\rho_{2}, \lambda_{2}}^{\sigma_{2}}\left(\ell_{4}-\ell_{3}\right)}{4} \\
& \times\left\{\int_{0}^{1} \int_{0}^{1} t^{\alpha} r^{\beta}\left|\frac{\partial^{2} f}{\partial t \partial r}\left(\ell_{1}+t \mathcal{F}_{\rho_{1}, \lambda_{1}}^{\sigma_{1}}\left(\ell_{2}-\ell_{1}\right), \ell_{3}+r \mathcal{F}_{\rho_{2}, \lambda_{2}}^{\sigma_{2}}\left(\ell_{4}-\ell_{3}\right)\right)\right| d r d t\right. \\
+ & \int_{0}^{1} \int_{0}^{1}(1-t)^{\alpha} r^{\beta}\left|\frac{\partial^{2} f}{\partial t \partial r}\left(\ell_{1}+t \mathcal{F}_{\rho_{1}, \lambda_{1}}^{\sigma_{1}}\left(\ell_{2}-\ell_{1}\right), \ell_{3}+r \mathcal{F}_{\rho_{2}, \lambda_{2}}^{\sigma_{2}}\left(\ell_{4}-\ell_{3}\right)\right)\right| d r d t \\
+ & \int_{0}^{1} \int_{0}^{1} t^{\alpha}(1-r)^{\beta}\left|\frac{\partial^{2} f}{\partial t \partial r}\left(\ell_{1}+t \mathcal{F}_{\rho_{1}, \lambda_{1}}^{\sigma_{1}}\left(\ell_{2}-\ell_{1}\right), \ell_{3}+r \mathcal{F}_{\rho_{2}, \lambda_{2}}^{\sigma_{2}}\left(\ell_{4}-\ell_{3}\right)\right)\right| d r d t \\
+ & \left.\int_{0}^{1} \int_{0}^{1}(1-t)^{\alpha}(1-r)^{\beta}\left|\frac{\partial^{2} f}{\partial t \partial r}\left(\ell_{1}+t \mathcal{F}_{\rho_{1}, \lambda_{1}}^{\sigma_{1}}\left(\ell_{2}-\ell_{1}\right), \ell_{3}+r \mathcal{F}_{\rho_{2}, \lambda_{2}}^{\sigma_{2}}\left(\ell_{4}-\ell_{3}\right)\right)\right| d r d t\right\} \\
\leq \frac{\mathcal{F}_{\rho_{1}, \lambda_{1}}^{\sigma_{1}}\left(\ell_{2}-\ell_{1}\right) \mathcal{F}_{\rho_{2}, \lambda_{2}}^{\sigma_{2}}\left(\ell_{4}-\ell_{3}\right)}{4} \times & \left\{\left(\int_{0}^{1} \int_{0}^{1} t^{p \alpha} r^{p \beta} d r d t\right)^{\frac{1}{p}}+\left(\int_{0}^{1} \int_{0}^{1}(1-t)^{p \alpha} r r^{p \beta} d r d t\right)^{\frac{1}{p}}\right. \\
& \left.+\left(\int_{0}^{1} \int_{0}^{1} t^{p \alpha}(1-r)^{p \beta} d r d t\right)^{\frac{1}{p}}+\left(\int_{0}^{1} \int_{0}^{1}(1-t)^{p \alpha}(1-r)^{p \beta} d r d t\right)^{\frac{1}{p}}\right\} \\
\leq & \frac{\mathcal{F}_{\rho_{1}, \lambda_{1}}^{\sigma_{1}}\left(\ell_{2}-\ell_{1}\right) \mathcal{F}_{\rho_{2}, \lambda_{2}}^{\sigma_{2}}\left(\ell_{4}-\ell_{3}\right)}{\sqrt[p]{(p \alpha+1)(p \beta+1)}} \\
& \left(\int_{0}^{1} \int_{0}^{1}\left|\frac{\partial^{2} f}{\partial t \partial r}\left(\ell_{1}+t \mathcal{F}_{\rho_{1}, \lambda_{1}}^{\sigma_{1}}\left(\ell_{2}-\ell_{1}\right), \ell_{3}+r \mathcal{F}_{\rho_{2}, \lambda_{2}}^{\sigma_{2}}\left(\ell_{4}-\ell_{3}\right)\right)\right|^{q} d r d t\right)^{\frac{1}{q}}
\end{aligned}
$$




$$
\begin{gathered}
\times\left[\int _ { 0 } ^ { 1 } \int _ { 0 } ^ { 1 } \left\{(1-t)(1-r)\left|\frac{\partial^{2} f}{\partial t \partial r}\left(\ell_{1}, \ell_{3}\right)\right|^{q}+t(1-r)\left|\frac{\partial^{2} f}{\partial t \partial r}\left(\ell_{2}, \ell_{3}\right)\right|^{q}\right.\right. \\
\left.\left.+(1-t) r\left|\frac{\partial^{2} f}{\partial t \partial r}\left(\ell_{1}, \ell_{4}\right)\right|^{q}+t r\left|\frac{\partial^{2} f}{\partial t \partial r}\left(\ell_{2}, \ell_{4}\right)\right|^{q}\right\} d r d t\right]^{\frac{1}{q}} \\
=\frac{\mathcal{F}_{\rho_{1}, \lambda_{1}}^{\sigma_{1}}\left(\ell_{2}-\ell_{1}\right) \mathcal{F}_{\rho_{2}, \lambda_{2}}^{\sigma_{2}}\left(\ell_{4}-\ell_{3}\right)}{\sqrt[p]{(p \alpha+1)(p \beta+1)}} \\
\times \sqrt[q]{\frac{\left|\frac{\partial^{2} f}{\partial t \partial r}\left(\ell_{1}, \ell_{3}\right)\right|^{q}+\left|\frac{\partial^{2} f}{\partial t \partial r}\left(\ell_{2}, \ell_{3}\right)\right|^{q}+\left|\frac{\partial^{2} f}{\partial t \partial r}\left(\ell_{1}, \ell_{4}\right)\right|^{q}+\left|\frac{\partial^{2} f}{\partial t \partial r}\left(\ell_{2}, \ell_{4}\right)\right|^{q}}{4}} .
\end{gathered}
$$

The proof is complete.

We point out some special cases of Theorem 3.

Corollary 1. Choosing $p=q=2$ in Theorem 3, we get:

$$
\begin{aligned}
& \left|E_{f}\left(\alpha, \beta, \ell_{1}, \ell_{2}, \ell_{3}, \ell_{4} ; \mathcal{F}_{\rho_{1}, \lambda_{1}}^{\sigma_{1}}, \mathcal{F}_{\rho_{2}, \lambda_{2}}^{\sigma_{2}}\right)\right| \leq \frac{\mathcal{F}_{\rho_{1}, \lambda_{1}}^{\sigma_{1}}\left(\ell_{2}-\ell_{1}\right) \mathcal{F}_{\rho_{2}, \lambda_{2}}^{\sigma_{2}}\left(\ell_{4}-\ell_{3}\right)}{\sqrt{(2 \alpha+1)(2 \beta+1)}} . \\
& \quad \times \sqrt{\frac{\left|\frac{\partial^{2} f}{\partial t \partial r}\left(\ell_{1}, \ell_{3}\right)\right|^{2}+\left|\frac{\partial^{2} f}{\partial t \partial r}\left(\ell_{2}, \ell_{3}\right)\right|^{2}+\left|\frac{\partial^{2} f}{\partial t \partial r}\left(\ell_{1}, \ell_{4}\right)\right|^{2}+\left|\frac{\partial^{2} f}{\partial t \partial r}\left(\ell_{2}, \ell_{4}\right)\right|^{2}}{4}} .
\end{aligned}
$$

Corollary 2. Choosing $\mathcal{F}_{\rho_{1}, \lambda_{1}}^{\sigma_{1}}\left(\ell_{2}-\ell_{1}\right)=\ell_{2}-\ell_{1}$ and $\mathcal{F}_{\rho_{2}, \lambda_{2}}^{\sigma_{2}}\left(\ell_{4}-\ell_{3}\right)=\ell_{4}-\ell_{3}$ in Theorem 3 , we have:

$$
\begin{gathered}
\left|E_{f}\left(\alpha, \beta, \ell_{1}, \ell_{2}, \ell_{3}, \ell_{4}\right)\right| \leq \frac{\left(\ell_{2}-\ell_{1}\right)\left(\ell_{4}-\ell_{3}\right)}{\sqrt[p]{(p \alpha+1)(p \beta+1)}} \\
\times \sqrt[q]{\frac{\left|\frac{\partial^{2} f}{\partial t \partial r}\left(\ell_{1}, \ell_{3}\right)\right|^{q}+\left|\frac{\partial^{2} f}{\partial t \partial r}\left(\ell_{2}, \ell_{3}\right)\right|^{q}+\left|\frac{\partial^{2} f}{\partial t \partial r}\left(\ell_{1}, \ell_{4}\right)\right|^{q}+\left|\frac{\partial^{2} f}{\partial t \partial r}\left(\ell_{2}, \ell_{4}\right)\right|^{q}}{4}} .
\end{gathered}
$$

Corollary 3. Taking $\left|\frac{\partial^{2} f}{\partial t \partial r}\right| \leq K$ in Theorem 3, we obtain:

$$
\left|E_{f}\left(\alpha, \beta, \ell_{1}, \ell_{2}, \ell_{3}, \ell_{4} ; \mathcal{F}_{\rho_{1}, \lambda_{1}}^{\sigma_{1}}, \mathcal{F}_{\rho_{2}, \lambda_{2}}^{\sigma_{2}}\right)\right| \leq K \frac{\mathcal{F}_{\rho_{1}, \lambda_{1}}^{\sigma_{1}}\left(\ell_{2}-\ell_{1}\right) \mathcal{F}_{\rho_{2}, \lambda_{2}}^{\sigma_{2}}\left(\ell_{4}-\ell_{3}\right)}{\sqrt[p]{(p \alpha+1)(p \beta+1)}} .
$$

Corollary 4. Choosing $\mathcal{F}_{\rho_{1}, \lambda_{1}}^{\sigma_{1}}\left(\ell_{2}-\ell_{1}\right)=\ell_{2}-\ell_{1}$ and $\mathcal{F}_{\rho_{2}, \lambda_{2}}^{\sigma_{2}}\left(\ell_{4}-\ell_{3}\right)=\ell_{4}-\ell_{3}$ in Corollary 3 , we get:

$$
\left|E_{f}\left(\alpha, \beta, \ell_{1}, \ell_{2}, \ell_{3}, \ell_{4}\right)\right| \leq K \frac{\left(\ell_{2}-\ell_{1}\right)\left(\ell_{4}-\ell_{3}\right)}{\sqrt[p]{(p \alpha+1)(p \beta+1)}} .
$$

Theorem 4. Let $f: \Delta \subset \mathbb{R}^{2} \longrightarrow \mathbb{R}$ be a partial differentiable function on $\Delta$ with $\mathcal{F}_{\rho_{1}, \lambda_{1}}^{\sigma_{1}}\left(\ell_{2}-\ell_{1}\right)>0$ and $\mathcal{F}_{\rho_{2}, \lambda_{2}}^{\sigma_{2}}\left(\ell_{4}-\ell_{3}\right)>0$ and $\frac{\partial^{2} f}{\partial t \partial r} \in L_{1}(\Delta)$. If $\left|\frac{\partial^{2} f}{\partial t \partial r}\right|^{q}$ is a generalized coordinated $\phi$-convex function where $q \geq 1$, then the following inequality holds:

$$
\begin{aligned}
& \left|E_{f}\left(\alpha, \beta, \ell_{1}, \ell_{2}, \ell_{3}, \ell_{4} ; \mathcal{F}_{\rho_{1}, \lambda_{1}}^{\sigma_{1}}, \mathcal{F}_{\rho_{2}, \lambda_{2}}^{\sigma_{2}}\right)\right| \\
& \quad \leq \frac{\mathcal{F}_{\rho_{1}, \lambda_{1}}^{\sigma_{1}}\left(\ell_{2}-\ell_{1}\right) \mathcal{F}_{\rho_{2}, \lambda_{2}}^{\sigma_{2}}\left(\ell_{4}-\ell_{3}\right)}{4}\left(\frac{1}{(\alpha+1)(\beta+1)}\right)^{1-\frac{1}{q}} \times
\end{aligned}
$$




$$
\sqrt[q]{\Psi(\alpha, \beta)\left\{\left|\frac{\partial^{2} f}{\partial t \partial r}\left(\ell_{1}, \ell_{3}\right)\right|^{q}+\left|\frac{\partial^{2} f}{\partial t \partial r}\left(\ell_{2}, \ell_{3}\right)\right|^{q}+\left|\frac{\partial^{2} f}{\partial t \partial r}\left(\ell_{1}, \ell_{4}\right)\right|^{q}+\left|\frac{\partial^{2} f}{\partial t \partial r}\left(\ell_{2}, \ell_{4}\right)\right|^{q}\right\}},
$$

where

$$
\Psi(\alpha, \beta)=\frac{2(\alpha+\beta+2)+\alpha \beta}{(\alpha+1)(\alpha+2)(\beta+1)(\beta+2)} .
$$

Proof. Using Lemma 1, the fact that $\left|\frac{\partial^{2} f}{\partial t \partial r}\right|^{q}$ is a generalized coordinated $\phi$-convex function, and the well-known power-mean inequality, we have:

$$
\begin{aligned}
& \left|E_{f}\left(\alpha, \beta, \ell_{1}, \ell_{2}, \ell_{3}, \ell_{4} ; \mathcal{F}_{\rho_{1}, \lambda_{1}}^{\sigma_{1}}, \mathcal{F}_{\rho_{2}, \lambda_{2}}^{\sigma_{2}}\right)\right| \\
& \leq \frac{\mathcal{F}_{\rho_{1}, \lambda_{1}}^{\sigma_{1}}\left(\ell_{2}-\ell_{1}\right) \mathcal{F}_{\rho_{2}, \lambda_{2}}^{\sigma_{2}}\left(\ell_{4}-\ell_{3}\right)}{4} \\
& \times\left\{\int_{0}^{1} \int_{0}^{1} t^{\alpha} r^{\beta}\left|\frac{\partial^{2} f}{\partial t \partial r}\left(\ell_{1}+t \mathcal{F}_{\rho_{1}, \lambda_{1}}^{\sigma_{1}}\left(\ell_{2}-\ell_{1}\right), \ell_{3}+r \mathcal{F}_{\rho_{2}, \lambda_{2}}^{\sigma_{2}}\left(\ell_{4}-\ell_{3}\right)\right)\right| d r d t\right. \\
& +\int_{0}^{1} \int_{0}^{1}(1-t)^{\alpha} r^{\beta}\left|\frac{\partial^{2} f}{\partial t \partial r}\left(\ell_{1}+t \mathcal{F}_{\rho_{1}, \lambda_{1}}^{\sigma_{1}}\left(\ell_{2}-\ell_{1}\right), \ell_{3}+r \mathcal{F}_{\rho_{2}, \lambda_{2}}^{\sigma_{2}}\left(\ell_{4}-\ell_{3}\right)\right)\right| d r d t \\
& +\int_{0}^{1} \int_{0}^{1} t^{\alpha}(1-r)^{\beta}\left|\frac{\partial^{2} f}{\partial t \partial r}\left(\ell_{1}+t \mathcal{F}_{\rho_{1}, \lambda_{1}}^{\sigma_{1}}\left(\ell_{2}-\ell_{1}\right), \ell_{3}+r \mathcal{F}_{\rho_{2}, \lambda_{2}}^{\sigma_{2}}\left(\ell_{4}-\ell_{3}\right)\right)\right| d r d t \\
& \left.+\int_{0}^{1} \int_{0}^{1}(1-t)^{\alpha}(1-r)^{\beta}\left|\frac{\partial^{2} f}{\partial t \partial r}\left(\ell_{1}+t \mathcal{F}_{\rho_{1}, \lambda_{1}}^{\sigma_{1}}\left(\ell_{2}-\ell_{1}\right), \ell_{3}+r \mathcal{F}_{\rho_{2}, \lambda_{2}}^{\sigma_{2}}\left(\ell_{4}-\ell_{3}\right)\right)\right| d r d t\right\} \\
& \leq \frac{\mathcal{F}_{\rho_{1}, \lambda_{1}}^{\sigma_{1}}\left(\ell_{2}-\ell_{1}\right) \mathcal{F}_{\rho_{2}, \lambda_{2}}^{\sigma_{2}}\left(\ell_{4}-\ell_{3}\right)}{4} \\
& \times\left\{\left(\int_{0}^{1} \int_{0}^{1} t^{\alpha} r^{\beta} d r d t\right)^{1-\frac{1}{q}}+\left(\int_{0}^{1} \int_{0}^{1}(1-t)^{\alpha} r^{\beta} d r d t\right)^{1-\frac{1}{q}}\right. \\
& \left.+\left(\int_{0}^{1} \int_{0}^{1} t^{\alpha}(1-r)^{\beta} d r d t\right)^{1-\frac{1}{q}}+\left(\int_{0}^{1} \int_{0}^{1}(1-t)^{\alpha}(1-r)^{\beta} d r d t\right)^{1-\frac{1}{q}}\right\} \\
& \times\left[\int_{0}^{1} \int_{0}^{1}\left\{t^{\alpha} r^{\beta}+(1-t)^{\alpha} r^{\beta}+t^{\alpha}(1-r)^{\beta}+(1-t)^{\alpha}(1-r)^{\beta}\right\}\right. \\
& \left.\times\left|\frac{\partial^{2} f}{\partial t \partial r}\left(\ell_{1}+t \mathcal{F}_{\rho_{1}, \lambda_{1}}^{\sigma_{1}}\left(\ell_{2}-\ell_{1}\right), \ell_{3}+r \mathcal{F}_{\rho_{2}, \lambda_{2}}^{\sigma_{2}}\left(\ell_{4}-\ell_{3}\right)\right)\right|^{q} d r d t\right]^{\frac{1}{q}} \\
& \leq \frac{\mathcal{F}_{\rho_{1}, \lambda_{1}}^{\sigma_{1}}\left(\ell_{2}-\ell_{1}\right) \mathcal{F}_{\rho_{2}, \lambda_{2}}^{\sigma_{2}}\left(\ell_{4}-\ell_{3}\right)}{4}\left(\frac{1}{(\alpha+1)(\beta+1)}\right)^{1-\frac{1}{q}} \\
& \times\left[\int_{0}^{1} \int_{0}^{1}\left\{t^{\alpha} r^{\beta}+(1-t)^{\alpha} r^{\beta}+t^{\alpha}(1-r)^{\beta}+(1-t)^{\alpha}(1-r)^{\beta}\right\}\right. \\
& \times\left\{(1-t)(1-r)\left|\frac{\partial^{2} f}{\partial t \partial r}\left(\ell_{1}, \ell_{3}\right)\right|^{q}+t(1-r)\left|\frac{\partial^{2} f}{\partial t \partial r}\left(\ell_{2}, \ell_{3}\right)\right|^{q}\right. \\
& \left.\left.+(1-t) r\left|\frac{\partial^{2} f}{\partial t \partial r}\left(\ell_{1}, \ell_{4}\right)\right|^{q}+t r\left|\frac{\partial^{2} f}{\partial t \partial r}\left(\ell_{2}, \ell_{4}\right)\right|^{q}\right\} d r d t\right]^{\frac{1}{q}} \\
& =\frac{\mathcal{F}_{\rho_{1}, \lambda_{1}}^{\sigma_{1}}\left(\ell_{2}-\ell_{1}\right) \mathcal{F}_{\rho_{2}, \lambda_{2}}^{\sigma_{2}}\left(\ell_{4}-\ell_{3}\right)}{4}\left(\frac{1}{(\alpha+1)(\beta+1)}\right)^{1-\frac{1}{q}} \times \\
& \sqrt[q]{\Psi(\alpha, \beta)\left\{\left|\frac{\partial^{2} f}{\partial t \partial r}\left(\ell_{1}, \ell_{3}\right)\right|^{q}+\left|\frac{\partial^{2} f}{\partial t \partial r}\left(\ell_{2}, \ell_{3}\right)\right|^{q}+\left|\frac{\partial^{2} f}{\partial t \partial r}\left(\ell_{1}, \ell_{4}\right)\right|^{q}+\left|\frac{\partial^{2} f}{\partial t \partial r}\left(\ell_{2}, \ell_{4}\right)\right|^{q}\right\}} \text {. }
\end{aligned}
$$


The proof of Theorem 4 is complete.

We point out some special cases of Theorem 4.

Corollary 5. Choosing $q=1$ in Theorem 4, we get:

$$
\begin{aligned}
& \left|E_{f}\left(\alpha, \beta, \ell_{1}, \ell_{2}, \ell_{3}, \ell_{4} ; \mathcal{F}_{\rho_{1}, \lambda_{1}}^{\sigma_{1}}, \mathcal{F}_{\rho_{2}, \lambda_{2}}^{\sigma_{2}}\right)\right| \\
& \leq \Psi(\alpha, \beta) \frac{\mathcal{F}_{\rho_{1}, \lambda_{1}}^{\sigma_{1}}\left(\ell_{2}-\ell_{1}\right) \mathcal{F}_{\rho_{2}, \lambda_{2}}^{\sigma_{2}}\left(\ell_{4}-\ell_{3}\right)}{4} \times \\
& \quad\left\{\left|\frac{\partial^{2} f}{\partial t \partial r}\left(\ell_{1}, \ell_{3}\right)\right|+\left|\frac{\partial^{2} f}{\partial t \partial r}\left(\ell_{2}, \ell_{3}\right)\right|+\left|\frac{\partial^{2} f}{\partial t \partial r}\left(\ell_{1}, \ell_{4}\right)\right|+\left|\frac{\partial^{2} f}{\partial t \partial r}\left(\ell_{2}, \ell_{4}\right)\right|\right\} .
\end{aligned}
$$

Corollary 6. Choosing $\mathcal{F}_{\rho_{1}, \lambda_{1}}^{\sigma_{1}}\left(\ell_{2}-\ell_{1}\right)=\ell_{2}-\ell_{1}$ and $\mathcal{F}_{\rho_{2}, \lambda_{2}}^{\sigma_{2}}\left(\ell_{4}-\ell_{3}\right)=\ell_{4}-\ell_{3}$ in Theorem 4, we have:

$$
\begin{aligned}
& \left|E_{f}\left(\alpha, \beta, \ell_{1}, \ell_{2}, \ell_{3}, \ell_{4}\right)\right| \leq \frac{\left(\ell_{2}-\ell_{1}\right)\left(\ell_{4}-\ell_{3}\right)}{4}\left(\frac{1}{(\alpha+1)(\beta+1)}\right)^{1-\frac{1}{q}} \times \\
& \sqrt[q]{\Psi(\alpha, \beta)\left\{\left|\frac{\partial^{2} f}{\partial t \partial r}\left(\ell_{1}, \ell_{3}\right)\right|^{q}+\left|\frac{\partial^{2} f}{\partial t \partial r}\left(\ell_{2}, \ell_{3}\right)\right|^{q}+\left|\frac{\partial^{2} f}{\partial t \partial r}\left(\ell_{1}, \ell_{4}\right)\right|^{q}+\left|\frac{\partial^{2} f}{\partial t \partial r}\left(\ell_{2}, \ell_{4}\right)\right|^{q}\right\}} .
\end{aligned}
$$

Corollary 7. Taking $\left|\frac{\partial^{2} f}{\partial t \partial r}\right| \leq K$ in Theorem 4, we obtain:

$$
\begin{aligned}
& \left|E_{f}\left(\alpha, \beta, \ell_{1}, \ell_{2}, \ell_{3}, \ell_{4} ; \mathcal{F}_{\rho_{1}, \lambda_{1}}^{\sigma_{1}}, \mathcal{F}_{\rho_{2}, \lambda_{2}}^{\sigma_{2}}\right)\right| \\
& \leq 4^{\frac{1-q}{q}} \mathcal{F}_{\rho_{1}, \lambda_{1}}^{\sigma_{1}}\left(\ell_{2}-\ell_{1}\right) \mathcal{F}_{\rho_{2}, \lambda_{2}}^{\sigma_{2}}\left(\ell_{4}-\ell_{3}\right)\left(\frac{1}{(\alpha+1)(\beta+1)}\right)^{1-\frac{1}{q}} \sqrt[q]{\Psi(\alpha, \beta)} .
\end{aligned}
$$

Corollary 8. Choosing $\mathcal{F}_{\rho_{1}, \lambda_{1}}^{\sigma_{1}}\left(\ell_{2}-\ell_{1}\right)=\ell_{2}-\ell_{1}$ and $\mathcal{F}_{\rho_{2}, \lambda_{2}}^{\sigma_{2}}\left(\ell_{4}-\ell_{3}\right)=\ell_{4}-\ell_{3}$ in Corollary 7 , we get:

$$
\left|E_{f}\left(\alpha, \beta, \ell_{1}, \ell_{2}, \ell_{3}, \ell_{4}\right)\right| \leq 4^{\frac{1-q}{q}}\left(\ell_{2}-\ell_{1}\right)\left(\ell_{4}-\ell_{3}\right)\left(\frac{1}{(\alpha+1)(\beta+1)}\right)^{1-\frac{1}{q}} \sqrt[q]{\Psi(\alpha, \beta)}
$$

Remark 3. Taking $\alpha=\beta=1$ in Theorems 2-4, we get some Hermite-Hadamard-type inequalities for the classical integral. Furthermore, for different positive values of $\rho_{1}, \rho_{2}, \lambda_{1}, \lambda_{2}>0$, where $\sigma_{1}=$ $\left(\sigma_{1}(0), \ldots, \sigma_{1}(k), \ldots\right)$ and $\sigma_{2}=\left(\sigma_{2}(0), \ldots, \sigma_{2}(k), \ldots\right)$ are bounded sequences of positive real numbers in our above results, we have different fascinating inequalities of the trapezium-type. The details are left to the interested reader.

\section{Conclusions}

In this paper, we defined a new class of functions, the so-called generalized coordinated $\phi$-convex involving Raina's functions and some Hermite-Hadamard-type integral inequalities ((6), (17), (22)) via the extended Riemann-Liouville fractional integral are provided as well. The interested reader can establish new inequalities via fractional operators or multiplicative integrals. Furthermore, given the usefulness of this type of integral inequalities and the integrals of fractional order in different areas of the pure and applied sciences, then the results presented can be applied in those investigations that require them. Similarly, the ideas considered in the development of this work are a contribution and stimulus for future research in the field of generalized convexity. 
Author Contributions: All the authors contributed equally to the compilation and revision of bibliographic references, the methodological development used in the proofs of the different theorems and propositions, and the writing and revision of the manuscript. All authors read and agreed to the published version of the manuscript.

Funding: This research was funded by Dirección de Investigación from Pontificia Universidad Católica del Ecuador in the research project entitled: Some integrals inequalities and generalized convexity (Algunas desigualdades integrales para funciones con algún tipo de convexidad generalizada y aplicaciones).

Acknowledgments: Miguel Vivas-Cortez thanks Dirección de Invstigación from Pontificia Universidad Católica del Ecuador, and Jorge E. Hernández Hernández thanks Consejo de Desarrollo Científico, Humanístico y Tecnológico from Universidad Centroccidental Lisandro Alvarado (Venezuela) for the technical support given in the development of the present article. Furthermore, all the authors thank the appointed referees for their appropriate comments in the evaluation of this work and the editorial team from Axioms for the serious and responsible work performed.

Conflicts of Interest: The authors declare no conflict of interest.

\section{References}

1. Bennett, C.; Sharpley, R. Interpolation of Operators; Academic Press: London, UK, 1998.

2. Ruel, J.J.; Ayres, M.P. Jensen's inequality predicts effects of environmental variation. Trends Ecol. Evol. 1999, 14, 361-366. [CrossRef]

3. Tomar, M.; Agarwal, P.; Jleli, M.; Samet, B. Certain Ostrowski type inequalities for generalized s-convex functions. J. Nonlinear Sci. Appl. 2017, 10, 5947-5957. [CrossRef]

4. Anderson, G.D.; Vamanamurthy, M.K.; Vuorinen, M. Generalized convexity and inequalities. J. Math. Anal. Appl. 2007, 335, 1294-1308. [CrossRef]

5. Grinalatt, M.; Linnainmaa, J.T. Jensen's Inequality, parameter uncertainty, and multiperiod investment. Rev. Asset Pricing Stud. 2011, 1, 1-34. [CrossRef]

6. Bracamonte, M.; Gimménez, J.; Vivas-Cortez, M.J. Hermite-Hadamard-Fejér type inequalities for strongly (s, m)-convex functions with modulus c, in second sense. Appl. Math. Inf. Sci. 2016, 10, 2045-2053. [CrossRef]

7. Dragomir, S.S.; Fitzpatrick, S. The Hadamard's inequality for s-convex functions in the second sense. Demonstr. Math. 1999, 32, 687-696.

8. Dragomir, S.S. On Hadamard's inequality for convex functions on the co-ordinates in a rectangle from the plane. Taiwan. J. Math. 2001, 4, 775-788. [CrossRef]

9. Dragomir, S.S. Inequalities of Hermite-Hadamard type for h-convex functions on linear spaces. Proyecc. J. Math. 2015, 32, 323-341. [CrossRef]

10. İscan, İ. Hermite-Hadamard type inequalities for harmonically convex functions. Hacet J. Math. Stats 2014, 43, 935-942. [CrossRef]

11. İscan, İ. Hermite-Hadamard type inequalities for harmonically $(\alpha, m)$-convex functions. arXiv 2015, arXiv:1307.5402v3.

12. İscan, İ. Ostrowski type inequalities for harmonically s-convex functions. Konuralp J. Math.2015, 3 , 63-74.

13. İscan, İ; Selim, N. Bekar, K. Hermite-Hadamard and Simpson type inequalities for differentiable harmonically P-functions. Br. J. Math. Comput. Sci. 2014, 4, 1908-1920.

14. Kashuri, A.; Liko, R. Some new fractional integral inequalities for generalized relative semi-(m- $(r, h 1, h 2)-$ preinvex mappings via generalized Mittag-Leffler function. Arab J. Math. Sci. 2019. [CrossRef]

15. Anastassiou, G.; Kashuri, A.; Liko, R. Local fractional integrals involving generalized strongly m-convex mappings. Arab. J. Math. 2019, 8, 95-107. [CrossRef]

16. Noor, M.A. Some new classes of non-convex functions. Nonlinear Funct. Anal. Appl. 2006, 11, $165-171$.

17. Vivas-Cortez, M.; García, C.; Kashuri, A.; Hernández Hernández, J.E. New Ostrowski Type Inequalities for Coordinated $(s, m)$-Convex Functions in the Second Sense. Appl. Math. Inf. Sci. 2019, 13, 821-829.

18. Vivas Cortez, M. Féjer type inequalities for (s, m)-convex functions in Second Sense. Appl. Math. Inf. Sci. 2016, 10, 1689-1696. [CrossRef]

19. Vivas-Cortez, M.; García, C. Ostrowski Type inequalities for functions whose derivatives are (m, h1, h2)convex. Appl. Math. Inf. Sci. 2017, 11, 79-86. [CrossRef]

20. Vivas, M.; Hernández Hernández, J.E.; Merentes, N. New Hermite-Hadamard and Jensen type inequalities for h-convex functions on fractal sets. Rev. Colomb. Mat. 2016, 50, 145-164. [CrossRef] 
21. Vivas Cortez, M. Relative strongly h-convex functions and integral inequalities. Appl. Math. Inf. Sci. Lett. 2016, 4, 39-45. [CrossRef]

22. Vivas-Cortez, M.J.; Kashuri, A.; Liko, R.; Hernández Hernández, J.E. Quantum estimates of Ostrowski inequalities for generalized $\phi$-convex functions. Symmetry 2019, 11, 1513. [CrossRef]

23. Rangel-Oliveros, Y.; Vivas-Cortez, M. Ostrowski type inequalities for functions whose second derivative are convex generalized. Appl. Math. Inf. Sci. 2018, 12, 1055-1064.

24. Vivas-Cortez, M.; Rangel-Oliveros, Y. An Inequality Related to s- $\phi-C o n v e x$ Functions. Appl. Math. Inf. Sci. $2020,14,151-154$.

25. Vivas-Cortez, M.J.; Kashuri, A.; Liko, R.; Hernández Hernández, J.E. Some Inequalities Using Generalized Convex Functions in Quantum Analysis. Symmetry 2019, 11, 1402. [CrossRef]

26. Jensen, J.L.W.V. Om konvexe Funktioner og Uligheder mellen Middelvaerdier. Nyt Tidsskr. Math. 1905 , 16, 49-69.

27. Jensen, J.L.W.V. Sur les fonctions convexes et les inegalités entre les valeurs moyennes. Acta Math. 1906, 30, 175-193. [CrossRef]

28. Hernández Hernández, J.E. Some fractional integral inequalities of Hermite Hadamard and Minkowski type. Sel. Mat. Univ. TRujillo Perú 2019, 6, 41-48.

29. Sambandham, S.; Vatsala, A.S. Basic Results for Sequential Caputo Fractional Differential Equations. Mathematics 2015, 3, 76-91. [CrossRef]

30. Awan, M.U.; Noor, M.A.; Mihai, M.V.; Noor, K.I.; Almohsen, B.A. Two dimensional extensions of Hermite-Hadamard's inequalities via preinvex functions. Rev. R. Acad. Cienc. Exactas Fìs. Nat. Ser. A Mat. 2019,113, 541-555. [CrossRef]

31. Sarikaya, M.Z. On the Hermite-Hadamard-type inequalities for co-ordinated convex function via fractional integrals. Integr. Transf. Spec. Func. 2014, 25, 134-147. [CrossRef]

32. Alomari, M.; Darus, M. The Hadamard's inequality for s-convex function of 2-variables on the coordinates. Int. J. Math. Anal. 2008, 2, 629-638.

33. Bai, S.P.; Qi, F. Some inequalities for $\left(s_{1}, m_{1}\right)-\left(s_{2}, m_{2}\right)$-convex functions on the co-ordinates. Glob. J. Math. Anal. 2013, 1, 22-28. [CrossRef]

34. Latif, M.A.; Dragomir, S.S. Some Hermite-Hadamard type inequalities for functions whose partial derivatives in absolute value are preinvex on the co-ordinates. Facta Univ. Ser. Math. Inf. 2013, 28, 257-270.

35. Mihai, M.V. New inequalities for co-ordinated convex functions via Riemann-Liouville fractional calculus. Tamkang J. Math. 2014, 45, 285-296.

36. Noor, M.A.; Noor, K.I.; Awan, M.U. Integral inequalities for coordinated harmonically convex functions. Complex Var. Elliptic Equat. 2015, 60, 776-786. [CrossRef]

37. Raina, R.K. On generalized Wright's hypergeometric functions and fractional calculus operators. East Asian Math. J. 2015, 21, 191-203.

38. Vivas-Cortez, M.J.; Liko, R.; Kashuri, A.; Hernández Hernández, J.E. New quantum estimates of trapezium-type inequalities for generalized $\phi$-convex functions. Mathematics 2019, 7, 1047. [CrossRef]

Publisher's Note: MDPI stays neutral with regard to jurisdictional claims in published maps and institutional affiliations.

(C) 2020 by the authors. Licensee MDPI, Basel, Switzerland. This article is an open access article distributed under the terms and conditions of the Creative Commons Attribution (CC BY) license (http:/ / creativecommons.org/licenses/by/4.0/). 